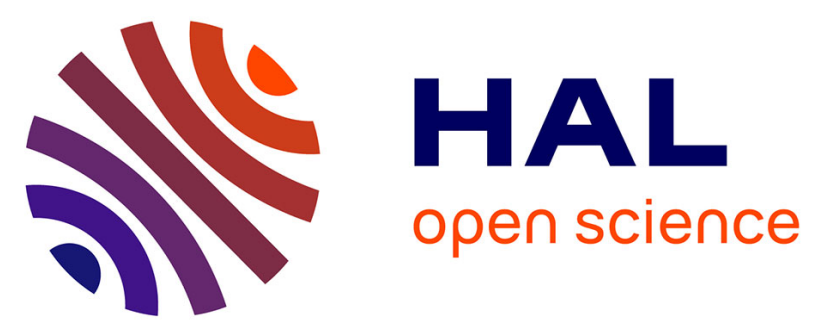

\title{
Null-collision meshless Monte-Carlo - A new reverse Monte-Carlo algorithm designed for laser-source emission in absorbing/scattering inhomogeneous media
} Morgan Sans, Mouna El-Hafi, V. Eymet, V. Forest, Richard A Fournier, N. Villefranque

\section{To cite this version:}

Morgan Sans, Mouna El-Hafi, V. Eymet, V. Forest, Richard A Fournier, et al.. Null-collision meshless Monte-Carlo - A new reverse Monte-Carlo algorithm designed for laser-source emission in absorbing/scattering inhomogeneous media. Journal of Quantitative Spectroscopy and Radiative Transfer, 2021, 271, pp.1-18/107725. 10.1016/j.jqsrt.2021.107725 . hal-03224186

\section{HAL Id: hal-03224186 \\ https://imt-mines-albi.hal.science/hal-03224186}

Submitted on 12 May 2021

HAL is a multi-disciplinary open access archive for the deposit and dissemination of scientific research documents, whether they are published or not. The documents may come from teaching and research institutions in France or abroad, or from public or private research centers.
L'archive ouverte pluridisciplinaire HAL, est destinée au dépôt et à la diffusion de documents scientifiques de niveau recherche, publiés ou non, émanant des établissements d'enseignement et de recherche français ou étrangers, des laboratoires publics ou privés. 


\title{
Null-collision meshless Monte-Carlo - A new reverse Monte-Carlo algorithm designed for laser-source emission in absorbing/scattering inhomogeneous media
}

\author{
M.Sans ${ }^{\mathrm{a}, *}$, M.El Hafi ${ }^{\mathrm{a}}$, V.Eymet ${ }^{\mathrm{b}}$, V.Forest ${ }^{\mathrm{b}}$, R.Fournier ${ }^{\mathrm{c}}$, N.Villefranque ${ }^{\mathrm{d}}$ \\ ${ }^{a}$ Université de Toulouse, Mines Albi, UMR 5302 - Centre de Recherche d'Albi en génie des Procédés des Solides Divisés, de l'Energie et de l'Environnement \\ (RAPSODEE), Campus Jarlard, F-81013, Albi CT cedex 09, France \\ ${ }^{b}$ Meso-Star SAS, Longages, France, www.meso-star.com \\ ${ }^{c}$ Université de Toulouse : UPS, INPT: LAPLACE (Laboratoire Plasma et ConversiondEnergie), 118 route de Narbonne, F-31062 Toulouse cedex 9, France. \\ ${ }^{d}$ Centre National de Recherches Météorologiques, UMR 3589 CNRS, Météo France, Toulouse, France
}

\begin{abstract}
Over recent decades, numerous studies in a myriad of research fields have improved the efficiency of the Monte-Carlo method to solve radiative transfers in heterogeneous media. The formalization of the concept of path integral formulation on which the construction of the random trajectories is based has made it possible to lay down a convenient framework to investigate sampling strategies and to design adapted low-variance algorithms. Our study focuses on the particular case of laser emission, which corresponds to a spatially-localized source emitting in a low solid angle, which partially illuminates the environment. In this case, the intrinsic characteristics of the laser emission cause problems of convergence with a Monte-Carlo method due to the difficulty in statistically linking sensors (probe points) to sources. This paper proposes, using integral formulation and a Null-Collision Algorithm (NCA), a practicable and simply implementable method to avoid such constraints. The intensity is broken down into a direct and a scattered term (local estimate technique). Then, a reworking of the various integral terms makes it possible to propose a complete algorithm adapted to a collimated source partially illuminating the studied scene. Non-zero contributions are brought more continuously to the Monte-Carlo weight and variance is strongly reduced. The entire methodology, from integral formulation to algorithmic interpretation, is presented step by step. For validation purposes, a new reverse and optimized Monte-Carlo algorithm is compared with an analogous Monte-Carlo for estimation of flux absorbed by a wall in an academic configuration, which ensures benchmark results. As the current proposed algorithm is highly suitable for building computer-generated images (probe calculation), the propagation of light due to laser emission through inhomogeneous environments is then illustrated by the construction of such images. This new tool provides useful support for experimental characterization of the radiative behaviour of particles.
\end{abstract}

Keywords: Monte-Carlo method, Null-Collision algorithm, Laser emission, Heterogeneous media, Radiative transfer, Computer-generated image

\section{Introduction}

Monte-Carlo Methods (MCMs) are algorithmic methods based on the probability theory. They are considered as reference methods as they provide a statistical uncertainty along with any estimate. MCMs are most efficient in highdimensional integral problems, complex geometries, and complex physics. They are used by many scientific communities (physics, economics, computer graphics...) who share similar needs in terms of computation, analysis and optimization, and face the same conceptual and methodological challenges. This forms a rich environment, where communities inspire each other, which does much to accelerate the methodological development of MCMs.

In the framework of radiative transfer, Monte-Carlo (MC) algorithms explicitly simulate the processes (emission, absorption, scattering, reflection) that model light propagation throughout a participating medium and its bounding surfaces. The analysis of the simulated paths provides important insights into the interaction of radiation with the medium. Many quantities can be estimated using different algorithms; from directional localized intensities (probe sensor) to spatially, hemispherically and/or spectrally integrated fluxes, to images (maps of intensity) as seen through imagers or human eyes.

Fundamentally, MC is a method that estimates integrals (and discrete sums) by interpreting them as expectations. Any quantity that can be expressed under an integral form can be estimated using a MC algorithm, including differential problems that are initially formulated outside the frame of stochastic processes $[1,2]$. The radiative-transfer equation (RTE) that models light propagation has a solution that is expressed as an integral formulation, which is recursive in scattering media. The quantity of interest is the incident intensity at a given location in a given direction $I_{0}=I\left(x_{0}, u_{0}\right)$, and the unknown in-scattering intensity at another location $x_{1}$ and in another direction $u_{1}$ appears under the integral. $I_{1}$ will in turn be evaluated by MC, following the same algorithm as for $I_{0} . I_{0}$ is hence written as 


\begin{tabular}{|c|c|c|c|}
\hline \multicolumn{4}{|c|}{ Nomenclature } \\
\hline \multicolumn{2}{|c|}{ Latin Symbols } & $\varphi$ & flux density $\left(\mathrm{W} \mathrm{m}^{-2} \mathrm{~nm}^{-1}\right)$ \\
\hline$I$ & radiant intensity $\left(\mathrm{W} \mathrm{m}^{-2} \mathrm{sr}^{-1} \mathrm{~Hz}^{-1}\right)$ & & \\
\hline & unit direction vector (-) & & \\
\hline $\mathbf{x}$ & coordinate vector $(\mathrm{m})$ & \multicolumn{2}{|c|}{ Subscripts } \\
\hline$p$ & probability density & $i$ & $i^{\text {th }}$ realisation of the Monte-Carlo algorithm \\
\hline & transmittance $(-)$ & $j$ & $j^{\text {th }}$ segment built \\
\hline $\mathcal{B}$ & boundary & & $k^{\text {th }}$ null-collision event \\
\hline$v$ & volume & & absorption \\
\hline $\mathcal{N}$ & volume of the laser sheet & & scattering \\
\hline $\mathcal{H}$ & Heaviside function & $\mathrm{g}$ & absorption, scattering or extinction \\
\hline $\mathcal{P}$ & probability density (test) & $\begin{array}{l}\mathrm{g} \\
\mathrm{n}\end{array}$ & null-collision \\
\hline$\hat{k}$ & uniform radiative coefficient & & wall \\
\hline$D$ & distance between laser sheet and the absorbing sur- & & intensity \\
\hline & face $(\mathrm{m})$ & & transmittance \\
\hline$d$ & distance travelled through the laser sheet & $\mathrm{a}+\mathrm{s}$ & extinction \\
\hline$e$ & thickness of the laser sheet (m) & & emitted \\
\hline$L$ & side length of the cube $(\mathrm{m})$ & & entry of the laser sheet \\
\hline & side length of the absorbing surface ( $\mathrm{x}$ direction) $(\mathrm{m})$ & $\max$ & maximum \\
\hline$l_{y}$ & side length of the absorbing surface (y direction) (m) & out & output of the laser sheet \\
\hline$P$ & total power $(\mathrm{W})$ & & \\
\hline & random variable & & \\
\hline & surface $\left(\mathrm{m}^{2}\right)$ & \multicolumn{2}{|c|}{ Superscripts } \\
\hline$w$ & Monte-Carlo weight & * & source \\
\hline & & & once-scattered \\
\hline \multicolumn{2}{|c|}{ Greek Symbols } & $\operatorname{dir}$ & direct \\
\hline$\delta$ & Dirac function & $\mathrm{s} 2$ & higher order of scattering \\
\hline & radiative property $\left(\mathrm{m}^{-1}\right)$ & scat & scattered \\
\hline$\Omega$ & solid angle (sr) & & \\
\hline & absorbtance (-) & & \\
\hline$\Delta \Omega$ & solid angle of dispersion of the laser source (sr) & \multicolumn{2}{|c|}{ Other Symbols } \\
\hline $\bar{\sigma}$ & Monte-Carlo weight-standard deviation & $\underline{\hat{X}}$ & $X$ computing using null-collision \\
\hline$\Phi$ & single-scattering phase function $(-)$ & & estimator of $X$ \\
\hline$\sigma$ & length $(\mathrm{m})$ & & preferential sampling of $X$ \\
\hline$\xi$ & transmittance along the radiative path (-) & AMC & Analogous Monte-Carlo \\
\hline$\rho$ & parameter of the choice of majorant (-) & ORMC & Optimized Reverse Monte-Carlo \\
\hline
\end{tabular}

the expectation of a function $f$ of the random variable $I_{1}$, itself written as the expectation of $f\left(I_{2}\right)$, and so on.

Because the expectation is a linear operator, only one sample of each random variable $I_{k}=I\left(x_{k}, u_{k}\right)$ is necessary to compute one sample of $I\left(x_{0}, u_{0}\right)$, as long as $f$ is a linear function:

$$
\mathbf{E}\left[f\left(\mathbf{E}\left[f\left(\ldots I_{k}\right)\right]\right)\right]=\mathbf{E}\left[f\left(f\left(\ldots I_{k}\right)\right)\right]
$$

More generally, combining unique samples of various random variables through linear functions to obtain one sample of the main variable, whose expectation is estimated, is called double randomization. This is a fundamental strength of MCMs.

One point of difficulty that has been much discussed in recent years with important implications for various communities, including the film industry, is the treatment of heterogeneous media. In the presence of spatial heterogeneities, the optical thick- ness $\tau$, which is the key variable of Beer's law of extinction, is in fact the integral of the varying extinction coefficient along the line of sight. This integral, in the context of MCMs, would be written as an expectation and a unique sample would be combined with other random variable samples, using double randomization. The difficulty is that this integral is combined nonlinearly with the rest of the integrals, through the exponential function. Because of this non-linearity, double randomization cannot be used, and the optical thickness must be computed deterministically along the whole path.

Fortunately, an unbiased way to bypass this non-linearity so that double randomization can be recovered exists: the nullcollision method. This method was known and used from the origins of MCMs, under different names in the different com- 
munities (Woodcock tracking [3], delta tracking [4], maximum cross-section [5]).

An intuitive interpretation of the method's principle is that transparent fictitious particles are added to the true medium to make it artificially homogeneous (cf. section 2.4). As the integral of extinction along the line of sight becomes a simple product, Beer's exponential can be easily inverted to sample the distance before next event. The resulting total medium is optically thicker than the original medium (the sampled distances are shorter); in compensation, some of the collision events are rejected: the so-called "null" collisions.

Historically, this method has been seen as a trick to avoid the heavy computation of optical thickness in heterogeneous media. It was only with the work of Galtier et al. [6] that the null-collision integral formulation was written and interpreted as a way of bypassing Beer's nonlinearity, giving birth to an entire family of designed and efficient algorithms and opening doors for extending MCMs to non-linear formulations [7, 8, 9]. In addition, the NCA allows a straightforward extension of the use of acceleration grids from surfaces to volumes. With the integral along the line of sight no longer appearing inside the integral formulation, acceleration strategies can be employed. Considering an artificially homogeneous medium, distance before next event can be sampled independently of the true radiative property field or without having to resort to the use of a deterministic method, freeing it from the dependence on resolution. However, the introduction of fictitious colliders can lead to an increase in the cost of calculation. This is particularly true for media with large spatial variations in radiative properties. The time spent processing null-collisions can become lengthy in the most transparent areas of the domain due to the large amount of rejections to be treated. An adapted subdivision of the field of interest into a given number of homogeneous media by parts (bounding boxes or acceleration grids) thus appears to be a convenient manner to ensure fast access to a true collision, greatly reducing computation cost, and is naturally related to the practice of NCA. Thus, compromises on data resolution are no longer necessary and the resolution of radiative problems with large scale ratios can be considered. Villefranque et al.[10] showed that employing an adaptive grid based on an unitary upper-bound optical thickness criterion, a computing time can be achieved that is almost insensitive to the resolution of cloud fields.

Strictly following the RTE solution expressed as an integral formulation leads to the construction of unidirectional paths starting at the position/direction of interest (probe calculation) and then conducting successive sampling of the different random variables: distance before next event, nature of the event, scattered direction of propagation, ... Thus, the integral formulation naturally simulates light flowing from sensor to sources and employs so-called "reverse" or "backward" algorithms. However, it is common to encounter a practice that relies almost exclusively on images from transport physics with "packet photons", those launched according to the laws of emissions and which will then evolve in the environment following the construction of paths that are also unidirectional. Such so-called "direct" or "analogous" algorithms simulate light flowing from sources to sensor. With regard to the reciprocity principle, "direct" and "reverse" algorithms are strictly equivalent. A photon emitted in $\mathbf{x}_{1}$ in the direction $\mathbf{u}_{1}$ has the same probability of being absorbed in $\mathbf{x}_{2}$ as a photon emitted in $\mathbf{x}_{2}$ in the direction $-\mathbf{u}_{1}$ of being absorbed in $\mathbf{x}_{1}$. This satisfies, in steady-state, the second principle of thermodynamics. However, in terms of convergence issues, one may be led to favour one or the other of the direct and reverse visions. Indeed, if one followed a direct approach, the probability of a photon reaching a small detector in a small solid angle is low. It is even null if the detector is punctual. Reverse algorithms are, in this case, well adapted. Reciprocally, if the sources are of small spatial and/or directional extent, then "direct" algorithms are more suitable. The choice between direct or reverse allows the optical paths of interest to be preferentially selected, according to the source/detector properties.

More generally, even if a direct algorithm is applicable, the integral formulation associated with reverse algorithms makes it possible to design adapted low-variance algorithms. Depending on which terms of the expectation (integral formulation) are interpreted as probability density functions (pdf) or as weights, different sampling strategies can be implemented, yielding different convergence properties. For example, the distance before next event is often sampled using the (normalized) exponential transmissivity directly as a pdf, which yields an exponentially distributed sample of distances, favouring short distances that contribute most to the path's weight.

In reality, any pdf can be used to sample any random event, as long as the sample's weight is corrected by the true probability of the event:

$$
\int_{\Gamma} \mathrm{d} x p(x) w_{x}=\int_{\Gamma} \mathrm{d} x g(x) \frac{w_{x} p(x)}{g(x)}
$$

Designing pdfs that will minimize the sample's variance is a very common practice in MCMs development, and is known as importance sampling [11].

Integral formulations are fundamental tools in the process of developing new algorithms that will be efficient in problematic cases where existing algorithms are slow to reach convergence: they provide a rigorous framework that guarantees flexibility and confidence. Because the integral directly translates into its associated algorithm, the numerical convergence of the algorithm can be improved by working on the integral.

An example of a difficulty that was bypassed by working from the integral formulation is when both sensor and sources are of small spatial and/or directional extent, resulting in a small shape factor. In these situations, most simulated paths do not connect sensor to lights, therefore not contributing to the input of energy measured at the sensor. Sampling enough paths to have a significant number of non-zero contributions can take a very long time. In the limit case when the lights are of null support in space or direction, no unidirectional path will ever bring a positive contribution to the estimated quantity, and "unidirectional" MCMs (direct or reverse) become impracticable.

In the framework of integral formulations such as the RTE, a solution is to split the observable into two contributions : a direct and an at-least-once-scattered term. The two terms of the 
sum are evaluated by MC: the direct contribution is evaluated by tracing a ray towards the source and is added to the total path's weight, while the other term is evaluated by continuing the path to the next scattering event (in the volume or at a surface). At each change of direction along the simulated "main" path, the intensity that needs to be estimated to continue the recursive path is, again, split into direct and at-least-once scattered. The final path's weight is a sum of contributions from paths of increasing order of scattering, emitted by the source and propagated along sub-paths of the main path. Reciprocally, with a direct algorithm, the path start from the source and the direct contribution is evaluated by simply tracing a ray towards the detector. Such techniques bear different names in the different communities in which they were developed; for instance the local estimate in atmospheric radiative transfer [5] or the nextevent estimation in neutron transport [12].

The method described above works only under certain conditions: the light source must either cover a large area or emit within a wide solid angle. For example, in solar radiation through the atmosphere, since the sun is modeled as a collimated source that illuminates the upper boundary of the atmosphere, direct contributions are evaluated by tracing a ray in the direction of incoming sunlight. Direct contributions from a localized spotlight are evaluated by tracing rays that link the current position to the light source. Indeed, this is only possible because sources such as the sun completely illuminate the environment. However, sources such as laser beams are highly localized in both space and direction. Hence, to address additional convergence challenges, an optimized specific algorithm is needed to propagate this type of source to a localized, collimated sensor.

In the engineering physics community, the numerical investigation of light transport by laser emission through heterogeneous media is a scientific challenge that can provide benefits for a wide variety of research fields, such as combustion, material characterization, biology, astrophysics or atmospheric radiative transfer. To the best of our knowledge, published attempts have thus far been limited to homogeneous media, such as Daun et al.'s [13] reverse MCM that numerically reproduces LaserInduced Incandescence (LII) measurements useful in the context of soot characterization. However, in such experiments, the medium emits within the laser sheet in all directions and and it is not necessary to treat the directional aspect of the laser source.

Different integral frameworks lead to different strategies to handle the same problems. For example, Veach's path integral formalism used in computer graphics [14] is slightly different from Feynman's path integral used in physics. Instead of successively sampling the events, the random variable is the ensemble of positions (vertices) that form the geometrical path. The challenge is to sample the paths efficiently according to the distribution of their total contributions: paths bringing large amounts of energy should be preferentially sampled. This more global interpretation of the path integral leads to different variance reduction techniques. Novak and colleagues' review article [4] provides referenced descriptions of some advanced techniques used in computer graphics.
Techniques to handle laser-like sources in heterogeneous media exist in the framework of Veach's path integral; however, importance sampling of joint random variables is necessary to ensure fast convergence (see, for instance, the line-to-line integration used in the context of Virtual Light Rays [4]). This can be quite complicated when the phase functions are peaked, as, for example, is the case in soots and clouds. Another limitation is that algorithms based on path integrals are often optimized using the general framework of multiple importance sampling [14], a technique that is not directly compatible with null-collision algorithms (see discussions in [4]).

Seeking solutions adapted to the path integral formalism of physicists, we worked at extending null-collision recursive integral formulations to efficiently handle laser-like sources, which partially illuminate the studied scene, and propose a complete methodology to compute the intensity reaching a punctual detector.

The following two sections (section 2 and section 3) describe the construction of the resulting algorithm, which is given in section 4. In section 2, the medium of propagation is described, the RTE is recalled, the MC estimator is presented, the nullcollision method is applied, and the algorithm that serves to evaluate transmissivity between two points is given. The proposition that specifically concerns laser beam emission is in section 3: the intensity is split into three terms representing contributions from a direct, a once-scattered and a more-than-once scattered path. Algorithms to compute each term are described, along with a variance reduction technique to sample a scattering point within the laser beam. In section 5, the new complete practicable and generic algorithm is validated by comparison with a well-mastered analog forward MC. For the sake of clarity, the passage between the integral formulation towards the application of null-collisions and the algorithmic interpretation is re-specified whenever necessary.

\section{Null-collision algorithms for radiative transfer}

\subsection{Configuration of interest, problematic and improvements proposed}

For validation purposes and as an illustration of the radiative problem needing to be solved, an academic test case is considered here (cf. fig. 1 a)). The idea is to compute the incident intensity on the detector (represented in blue) of size $l_{x} \times l_{y}$ with only a spatially localized source (size $L \times e$ ) which emits in a single direction (represented with red arrows). Thus, the direct propagation of the source (laser sheet) takes the geometrical form of a parallelepiped (represented in red) of size $L \times L \times e$. The thickness $e$ of the laser sheet is very low compared to others dimensions. The heterogeneous medium is defined by a heterogeneous radiative field inside the represented cube of side $L$ bounded by absorbing walls $\mathcal{B}$.

In this academic configuration, fig. 1 b) illustrates the difficulty of convergence encountered. In this figure, the relative statistical uncertainty $\bar{\sigma} / \bar{P}_{\mathrm{a}}$ of the estimated absorbed power $\bar{P}_{\mathrm{a}}$ as a function of the square sensor $\left(l_{\mathrm{x}}=l_{\mathrm{y}}=l_{\mathrm{d}}\right)$ size is represented for two different $\mathrm{MC}$ algorithms and for a given set of parameters. The analogous algorithm (result drawn in red) constructs 

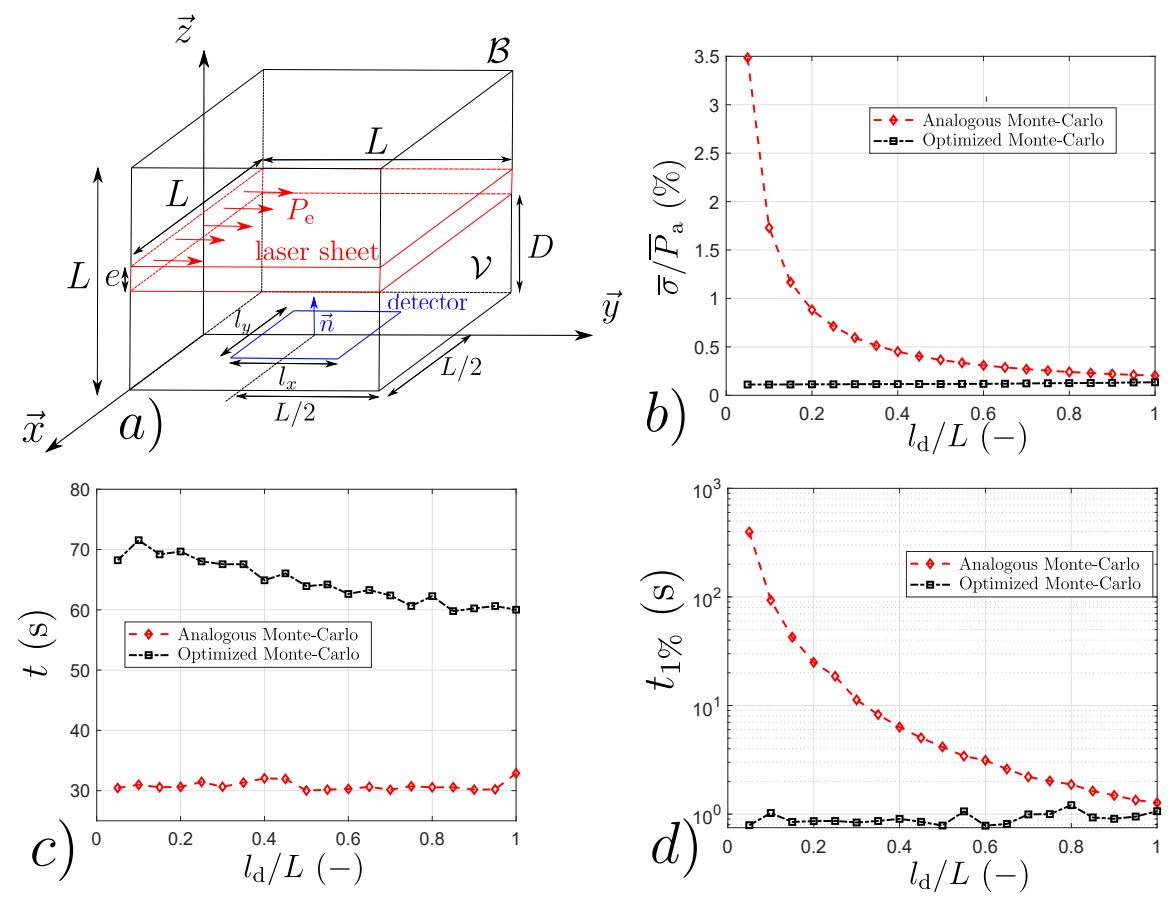

Figure 1: Considered problem: a) academic geometry b) convergence observed: relative variance of the estimator as function of detector size with $l_{\mathrm{x}}=l_{\mathrm{y}}=l_{\mathrm{d}}$ and for $10^{7}$ independent realizations c) computation time $t$ for $10^{7}$ independent realization as function of detector size c) computation time $t_{1} \%$ for a $1 \%$ statistical uncertainty as function of detector size

unidirectional paths departing from the emitting surface while the so-called "Optimized Monte-Carlo" (result drawn in black) corresponds to the new reverse algorithm detailed in this paper and based on paths initialized at the sensor and using a separation of the observable into direct/diffuse components.

In general, the direct and reverse algorithms make it possible to overcome both the spatial and directional constraints related to the source and the detector, respectively. However, when using unidirectional paths, the algorithm always depends on the difficulty to reach the second item. In this example, the source is null support in direction and of small spatial extent while the detector tends to be null support in space as the size of the sensor decreases. Using unidirectional paths, convergence is thus impossible with a reverse algorithm while a calculation is possible with an analogous MC algorithm. As shown in fig. $1 \mathrm{~b}$ ), statistical uncertainty of the AMC becomes more and more difficult to achieve as the size of the sensor decreases. For a given number of realizations (cf. fig. 1 c)), a Monte Carlo based on unidirectional paths is faster than the optimized Monte Carlo based on the construction of several subpaths. Nevertheless, as represented in fig. $1 \mathrm{~d}$ ), the computation time needed to obtain a good level of convergence of the AMC increases strongly with the decrease of the sensor size. In the asymptotic case of a spatially punctual sensor (null support), the calculation can not be performed as well. Hence, when it is a question of estimating the incident intensity on pixels (small spatial and directional extent), the AMC will also become impractical and an optimization of the whole algorithm is therefore necessary. Such optimization is provided and detailed in this paper.

In order to answer this question and produce computergenerated images while maintaining the use of null-collisions, it is convenient to use the framework made possible by integral formalism, which leads intuitively and naturally to reverse Monte-Carlo algorithms. Hence, in the following, only an optimized reverse MC algorithm is detailed, while a direct analogous MC algorithm is only used for validation purposes. Let us remark that, from the reverse formulation proposed, the building of the correspondent optimized and direct algorithm is straightforward. However, the choice between a direct and a reverse algorithm being strongly dependent on the studied configuration, this question is not discussed here.

The optimization presented in this paper makes it possible to avoid the constraints related to both a small directional and spatial extent of the second item (source or detector). In addition, as in the case of the academic configuration presented, the algorithm can also handle the case of null support in direction or space. Finally, as shown in fig. 1 b) and fig. $1 \mathrm{~d}$ ), once the computation has been made possible thanks to improvements in path construction, the variance is logically insensitive to size and direction constraints related to the second item (detector or source).

\subsection{Radiative Transfer}

The monochromatic intensity $I_{v}$ at a given position $\mathbf{x}$ and in a given direction $\mathbf{u}$ inside the semitransparent heterogeneous 
medium is a solution of the RTE:

$$
\begin{aligned}
\mathbf{u} \cdot \vec{\nabla} I_{\nu}(\mathbf{x}, \mathbf{u}) & =-\kappa_{\mathrm{a}, v}(\mathbf{x}) I_{v}(\mathbf{x}, \mathbf{u})-\kappa_{\mathrm{s}, v}(\mathbf{x}) I_{v}(\mathbf{x}, \mathbf{u}) \\
& +\frac{1}{4 \pi} \int_{\Omega^{\prime}=4 \pi} \kappa_{\mathrm{s}, v}(\mathbf{x}) \Phi_{\nu}\left(\mathbf{x}, \mathbf{u}^{\prime} \mid \mathbf{u}\right) I_{v}\left(\mathbf{x}, \overrightarrow{\mathbf{u}^{\prime}}\right) \mathrm{d} \mathbf{u}^{\prime}
\end{aligned}
$$

where $\kappa_{\mathrm{s} v}, \kappa_{\mathrm{a} v}$ and $\Phi_{v}$ are the monochromatic scattering, absorption and single scattering phase function, respectively. Let us note that the medium is assumed cold. Hence, the black-body emission term is not considered. The walls are assumed to be black and cold. No emission from walls is considered. In this problem, the laser is the only source present in the scene. Thus, the radiative boundary condition is written as:

$$
I_{\nu}\left(\mathbf{x}_{\mathrm{w}}, \mathbf{u}\right)=I_{v}^{*} \mathcal{H}\left(\mathbf{x} \in \mathcal{B}^{*} \cap \mathbf{u} \in \Omega^{*}\right)
$$

where $\mathcal{B}^{*}$ and $\Omega^{*}$ correspond to the domain where the energy is emitted and the direction of propagation, respectively. Thus, the intensity is non-zero only on the emission surface and in the direction of propagation. Otherwise, it is strictly null. In the following, the spectral index $v$ is removed for the sake of brevity but all considered quantities are monochromatic.

\subsection{Monte-Carlo method to solve radiative transfer}

The intensity at a given position $\mathbf{x}_{0}$, a given direction $\mathbf{u}_{0}$ and solution of the RTE (cf. eq. (1)) takes the following integral form:

$$
\begin{aligned}
I\left(\mathbf{x}_{0}, \mathbf{u}_{0}\right) & =\int_{0}^{\infty} \mathrm{d} \sigma_{1} \kappa_{\mathrm{s}}\left(\mathbf{x}_{0}-\sigma_{1} \mathbf{u}_{0}\right) \exp \left(-\int_{0}^{\sigma_{1}} \kappa_{\mathrm{a}+\mathrm{s}}\left(\mathbf{x}_{0}-\sigma_{1}^{\prime} \mathbf{u}_{0}\right) \mathrm{d} \sigma_{1}^{\prime}\right) \\
& \left.\times\left[\int_{4 \pi} \mathrm{d} \mathbf{u}_{1} \Phi\left(\mathbf{x}_{1}, \mathbf{u}_{0} \mid \mathbf{u}_{1}\right) I\left(\mathbf{x}_{1}, \mathbf{u}_{1}\right)\right)\right]
\end{aligned}
$$

where $\kappa_{\mathrm{a}+\mathrm{s}}=\kappa_{\mathrm{a}}+\kappa_{\mathrm{s}}$ the extinction coefficient and $\sigma_{1}$ all propagation distances before scattering along the line of sight. In other words, assuming no black-body emission from the medium, eq. (3) expresses the intensity at a given position $\mathbf{x}_{0}$ and a given direction $\mathbf{u}_{0}$ as a spatial integral of the incident radiant energy in-scattered at the position $\mathbf{x}_{1}=\mathbf{x}_{0}-\sigma_{1} \mathbf{u}_{0}$ and attenuated by extinction. Such an expression can be interpreted with a reverse $\mathrm{MC}$ algorithm.

By rearranging the previous equation, probability densities are brought up for algorithmic interpretation:

$\left.I\left(\mathbf{x}_{0}, \mathbf{u}_{0}\right)=\int_{0}^{\infty} \mathrm{d} \sigma_{1} p_{\Sigma}\left(\sigma_{1}\right) \mathcal{T}_{\mathrm{a}}\left(\mathbf{x}_{0}, \mathbf{x}_{1}\right) \int_{4 \pi} \mathrm{d} \mathbf{u}_{1} \Phi\left(\mathbf{x}_{1}, \mathbf{u}_{0} \mid \mathbf{u}_{1}\right) I\left(\mathbf{x}_{1}, \mathbf{u}_{1}\right)\right)$

where:

$$
p_{\Sigma}\left(\sigma_{1}\right)=\kappa_{\mathrm{S}}\left(\mathbf{x}_{0}-\sigma_{1} \mathbf{u}_{0}\right) \exp \left(-\int_{0}^{\sigma_{1}} \kappa_{\mathrm{S}}\left(\mathbf{x}_{0}-\sigma_{1}^{\prime} \mathbf{u}_{0}\right) \mathrm{d} \sigma_{1}^{\prime}\right)
$$

the probability density of a random variable $\Sigma$ corresponding to the distribution of scattering free path $\sigma_{1} \in[0 ;+\infty]$ in the direction $-\mathbf{u}_{0}$. The transmittance due only to absorption is defined as:

$$
\mathcal{T}_{\mathrm{a}}\left(\mathbf{x}_{0}, \mathbf{x}_{1}\right)=\exp \left(-\int_{0}^{\sigma_{1}} \kappa_{\mathrm{a}}\left(\mathbf{x}_{0}-\sigma_{1}^{\prime} \mathbf{u}_{0}\right) \mathrm{d} \sigma_{1}^{\prime}\right)
$$

which quantifies the part of the radiation that has not been absorbed between the two scattering positions.

As long as the incident radiant energy at the new position $\mathbf{x}_{j+1}$ and in the new direction $\mathbf{u}_{j+1}$ remains unknown, the procedure is pursued, which corresponds to a recursive process. The corresponding algorithm is described in algorithm 1.

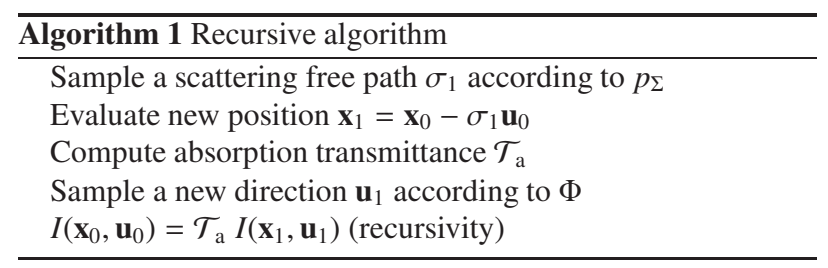

Scattering and absorption events are treated here separately through, firstly, the sampling of a scattering free path and, secondly, computation of a transmittance by absorption. In this field, a wide number of techniques exist. Since the question of determining the relevance of one kind of sampling method compared to others is not central in this work, the interested reader can refer to [15], which gives a broad overview of the existing methods. The choice made comes simply from the fact that the sampling of a scattering length appears naturally during the formulation of the solution due to the absence of black-body emission of the medium. As shown in [16], the sampling used here is particularly well adapted to mainly scattering media.

\subsection{Null-collision principle}

In the case of an inhomogeneous environment, the extinction coefficient, which varies along the line of sight, induces a nonlinearity of Beer's law. This makes it necessary to calculate the optical thickness in a deterministic way. Recent methodological developments [6] have shown that it is possible to use socalled null-collision MCM to avoid the latter constraint. The main idea is to add transparent fictitious collision, defined by $\kappa_{\mathrm{n}}=\hat{k}-\kappa$, the null-collision coefficient, in order to consider an artificially homogeneous medium. Thus, the resulting considered medium is optically thicker and some of the collisions are rejected by applying a purely forward scattering. For more details, the interested reader may refer to the recent work of El Hafi et al.[9] where the principle of similarity with a homogeneous medium is developed. The introduction of fictitious collision does not induce bias on the estimated value. It consists in adding $-\kappa_{\mathrm{n}} I(\mathbf{x}, \mathbf{u})+\int_{4 \pi} \kappa_{\mathrm{n}} I(\mathbf{x}, \mathbf{u}) \delta\left(\mathbf{x}, \mathbf{u}-\mathbf{u}^{\prime}\right)$ to the right-hand side of the RTE (cf. eq. (1)). The Dirac distribution $\delta$ ensures that the modified RTE has exactly the same solution:

$$
\begin{aligned}
\mathbf{u} \cdot \vec{\nabla} I(\mathbf{x}, \mathbf{u}) & =-\left(\kappa_{\mathrm{a}}(\mathbf{x})+\kappa_{\mathrm{S}}(\mathbf{x})+\kappa_{\mathrm{n}}\right) I(\mathbf{x}, \mathbf{u}) \\
& +\frac{1}{4 \pi} \int_{\Omega^{\prime}=4 \pi} \kappa_{\mathrm{S}}(\mathbf{x}) \Phi\left(\mathbf{x}, \mathbf{u}^{\prime} \mid \mathbf{u}\right) I\left(\mathbf{x}, \overrightarrow{\mathbf{u}^{\prime}}\right) \mathrm{d} \mathbf{u}^{\prime} \\
& +\int_{\Omega^{\prime}=4 \pi} \kappa_{\mathrm{n}} I(\mathbf{x}, \mathbf{u}) \delta\left(\mathbf{x}, \mathbf{u}-\mathbf{u}^{\prime}\right)
\end{aligned}
$$

The absorption and scattering being treated separately, a uniform scattering coefficient $\hat{k}_{\mathrm{s}}=\kappa_{\mathrm{s}}+\kappa_{\mathrm{n}}$ is defined. Likewise, a uniform absorbing coefficient $\hat{k}_{\mathrm{a}}=\kappa_{\mathrm{a}}+\kappa_{\mathrm{n}}$ is used to compute 
the transmittance by absorption with a null-collision method $\hat{\mathcal{T}}_{\mathrm{a}}\left(\mathbf{x}_{0}, \mathbf{x}_{1}\right)$. This specific point is the topic of the following section (cf. section 2.5). Let us note that the null-collision coefficient is always chosen as an upper bound of the true radiative field. However, the interested reader can find an extension to negative values of the null-collision coefficient in [6].

The formal solution of the modified RTE (eq. (7)) is:

$$
\begin{aligned}
& I\left(\mathbf{x}_{0}, \mathbf{u}_{0}\right)=\int_{0}^{\infty} \mathrm{d} \sigma_{1} \exp \left(-\int_{0}^{\sigma_{1}} \hat{k}_{\mathrm{s}} \mathrm{d} \sigma_{1}^{\prime}\right) \hat{\mathcal{T}}_{\mathrm{a}}\left(\mathbf{x}_{0}, \mathbf{x}_{1}\right) \\
& \times\left\{\begin{array}{c}
\kappa_{\mathrm{s}}\left(\mathbf{x}_{1}\right) \int_{4 \pi} \mathrm{d} \mathbf{u}_{1} \Phi\left(\mathbf{x}_{1}, \mathbf{u}_{0} \mid \mathbf{u}_{1}\right) I\left(\mathbf{x}_{1}, \mathbf{u}_{1}\right) \\
+\kappa_{\mathrm{n}}\left(\mathbf{x}_{1}\right) \int_{4 \pi} \mathrm{d} \mathbf{u}_{1} \delta\left(\mathbf{x}_{1}, \mathbf{u}_{1}-\mathbf{u}_{0}\right) I\left(\mathbf{x}_{1}, \mathbf{u}_{1}\right)
\end{array}\right\}
\end{aligned}
$$

which can be rewritten as:

$$
\begin{aligned}
& I\left(\mathbf{x}_{0}, \mathbf{u}_{0}\right)=\int_{0}^{\infty} \mathrm{d} \sigma_{1} \hat{k}_{\mathrm{s}} \exp \left(-\hat{k}_{\mathrm{s}} \sigma_{1}\right) \hat{\mathcal{T}}_{\mathrm{a}}\left(\mathbf{x}_{0}, \mathbf{x}_{1}\right) \\
& \times\left\{\begin{array}{c}
\frac{\kappa_{\mathrm{s}}\left(\mathbf{x}_{1}\right)}{\hat{k}_{\mathrm{s}}} \int_{4 \pi} \mathrm{d} \mathbf{u}_{1} \Phi\left(\mathbf{x}_{1}, \mathbf{u}_{0} \mid \mathbf{u}_{1}\right) I\left(\mathbf{x}_{1}, \mathbf{u}_{1}\right) \\
+\frac{\kappa_{\mathrm{n}}\left(\mathbf{x}_{1}\right)}{\hat{k}_{\mathrm{s}}} \int_{4 \pi} \mathrm{d} \mathbf{u}_{1} \delta\left(\mathbf{x}_{1}, \mathbf{u}_{1}-\mathbf{u}_{0}\right) I\left(\mathbf{x}_{1}, \mathbf{u}_{1}\right)
\end{array}\right\}
\end{aligned}
$$

The different probability densities are brought up:

$$
\begin{aligned}
& I\left(\mathbf{x}_{0}, \mathbf{u}_{0}\right)=\int_{0}^{\infty} \hat{p}_{\Sigma}\left(\sigma_{1}\right) \hat{\mathcal{T}}_{\mathrm{a}}\left(\mathbf{x}_{0}, \mathbf{x}_{1}\right) \\
& \times\left\{\begin{array}{c}
\mathcal{P}_{\mathrm{s}}\left(\mathbf{x}_{1}\right) \int_{4 \pi} \mathrm{d} \mathbf{u}_{1} \Phi\left(\mathbf{x}_{1}, \mathbf{u}_{0} \mid \mathbf{u}_{1}\right) I\left(\mathbf{x}_{1}, \mathbf{u}_{1}\right) \\
+\mathcal{P}_{\mathrm{n}}\left(\mathbf{x}_{1}\right) \int_{4 \pi} \mathrm{d} \mathbf{u}_{1} \delta\left(\mathbf{x}_{1}, \mathbf{u}_{1}-\mathbf{u}_{0}\right) I\left(\mathbf{x}_{1}, \mathbf{u}_{1}\right)
\end{array}\right\}
\end{aligned}
$$

with $\mathcal{P}_{\mathrm{s}}=\kappa_{\mathrm{S}} / \hat{k}_{\mathrm{s}}$ the probability that the collision is real (scattering) and $\mathcal{P}_{\mathrm{n}}=\left(1-\mathcal{P}_{\mathrm{s}}\right)$ the probability that the collision is null. The determination of the type of collision is thus carried out thanks to a Bernoulli test.

One can note that the latter formulation is only valid for an infinite medium. For the sake of brevity, interaction with walls is voluntarily not presented to lighten the mathematical formalism. However, this does not lead to major difficulties. A test to determine whether or not a collision would occur at the boundary should be added. This particular point will be dealt with during the presentation of the complete generic algorithm in section 5 .

Hence, in the case of an infinite medium, the corresponding algorithm is described in algorithm 2 .

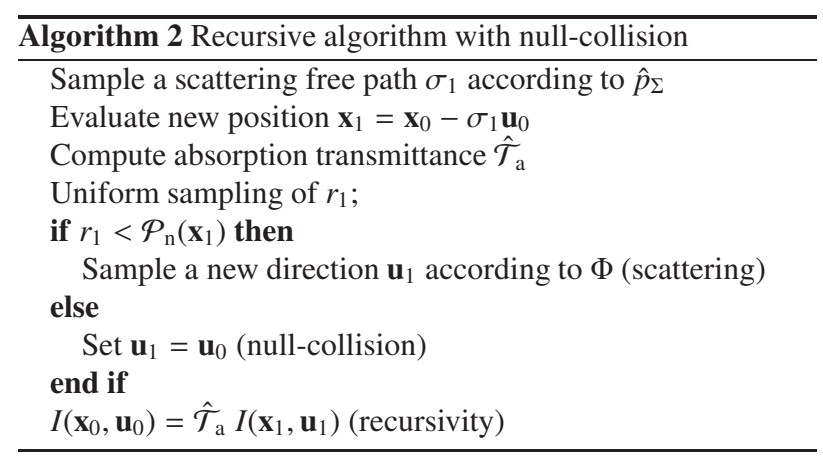

\subsection{Evaluation of a transmittance}

In our approach, we have to repeatedly calculate the transmittance either by absorption, scattering or extinction. This quantity represents the part of the radiative energy which crosses an arbitrary length $L$ in the unit direction $\mathbf{u}_{0}$ between two positions $\mathbf{x}_{0}$ and $\mathbf{x}_{1}$ of the semi-transparent medium:

$$
\mathcal{T}_{\mathrm{g}}(L)=\mathcal{T}_{\mathrm{g}}\left(\mathbf{x}_{0}, \mathbf{x}_{1}\right)=\exp \left(-\int_{0}^{L} \kappa_{\mathrm{g}}\left(\mathbf{x}_{0}-l_{1}^{\prime} \mathbf{u}_{0}\right) \mathrm{d} l_{1}^{\prime}\right)
$$

where $\kappa_{\mathrm{g}}$ can represent either the absorption, scattering or extinction coefficient. The index $g$ indicates the nature of the interaction between radiation and matter.

Let us introduce the quantity $p_{\mathcal{L}}\left(l_{1}\right) \mathrm{d} l_{1}$, which corresponds to the probability that a photon travels a distance greater than $l_{1}$ but smaller than $l_{1}+\mathrm{d} l_{1}$. According to eq. (11), we write:

$$
p_{\mathcal{L}}\left(l_{1}\right) \mathrm{d} l_{1}=\mathcal{T}_{\mathrm{g}}\left(l_{1}\right)-\mathcal{T}_{\mathrm{g}}\left(l_{1}+\mathrm{d} l_{1}\right)
$$

Hence, under a differential formalism:

$$
\begin{aligned}
p_{\mathcal{L}}\left(l_{1}\right) & =-\frac{\mathrm{d} \mathcal{T}_{\mathrm{g}}\left(l_{1}\right)}{\mathrm{d} l_{1}} \\
& =\kappa_{\mathrm{g}}\left(\mathbf{x}_{0}-l_{1} \mathbf{u}_{0}\right) \exp \left(-\int_{0}^{l_{1}} \kappa_{\mathrm{g}}\left(\mathbf{x}_{0}-l_{1}^{\prime} \mathbf{u}_{0}\right) \mathrm{d} l_{1}^{\prime}\right)
\end{aligned}
$$

the probability density corresponding to the distribution of a free path before either an absorption, scattering or extinction event is defined. A probability density (only for scattering) was previously used in eq. (5) to sample a scattering free path.

By appealing to the distribution of free paths $p_{\mathcal{L}}\left(l_{1}\right)$ as expressed in eq. (13), we can express the transmittance in eq. (11) with the following integral form:

$$
\begin{aligned}
\mathcal{T}_{\mathrm{g}}(L) & =\mathcal{T}_{\mathrm{g}}\left(\mathbf{x}_{0}, \mathbf{x}_{1}\right)=\int_{0}^{L} p_{\mathcal{L}}\left(l_{1}\right) \mathrm{d} l_{1} \\
& =\int_{0}^{\infty} p_{\mathcal{L}}\left(l_{1}\right) \mathcal{H}\left(l_{1}>L\right) \mathrm{d} l_{1}
\end{aligned}
$$

This integral form can be interpreted as a Monte-Carlo algorithm. It consists of sampling the free path according to $p_{\mathcal{L}}\left(l_{1}\right)$ (cf. eq. (13)) and checking if the distance travelled is smaller or greater than the targeted distance $L$. The MC weight $w_{\mathrm{T}}$ is zero if $l_{1}<L$ and unitary otherwise. An illustration of this procedure is proposed in fig. 2. 


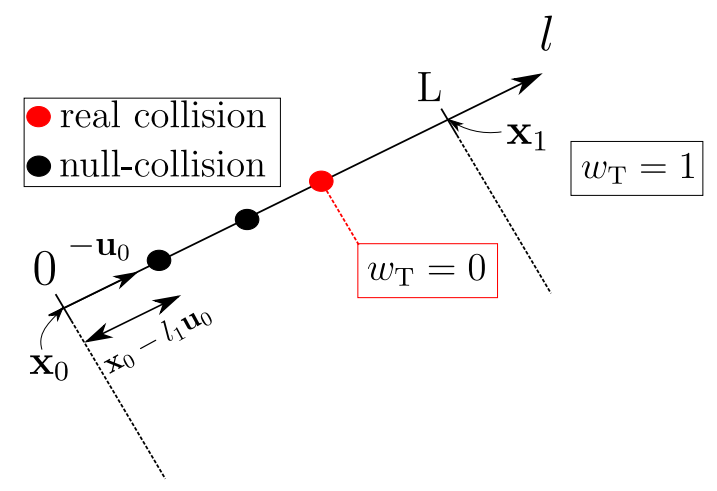

Figure 2: Computation of a transmittance using null-collision method: when a real collision is found, a test determines if the distance aimed for has been reached. The MC weight is zero if not and unitary otherwise

As explained above (cf. section 2.4), the use of the nullcollision method is a solution to bypass the Beer's non-linearity, which appears when considering a heterogeneous medium. The transmittance is then computed as:

$\hat{\mathcal{T}}_{\mathrm{g}}\left(\mathbf{x}_{0}, \mathbf{x}_{1}\right)=\int_{0}^{\infty} \hat{p}_{\mathcal{L}}\left(l_{1}\right) \mathrm{d} l_{1} \times$

$\left\{\begin{array}{l}\mathcal{H}\left(l_{1}>L\right) \times\{1\} \\ +H\left(l_{1}<L\right)\left[\frac{\kappa_{\mathrm{g}}\left(\mathbf{x}_{0}-l_{1} \mathbf{u}_{0}\right)}{\hat{k}_{\mathrm{g}}} \times\{0\}+\left(1-\frac{\kappa_{\mathrm{g}}\left(\mathbf{x}_{0}-l_{1} \mathbf{u}_{0}\right)}{\hat{k}_{\mathrm{g}}}\right)\left\{\hat{\mathcal{T}}_{\mathrm{g}}\left(\mathbf{x}_{0}-l_{1} \mathbf{u}_{0}, \mathbf{x}_{1}\right)\right\}\right.\end{array}\right.$

with $\hat{k}_{\mathrm{g}}=\kappa_{\mathrm{g}}+\kappa_{\mathrm{n}}$ the uniform field of absorption, scattering or extinction coefficient. The corresponding algorithm is given below:

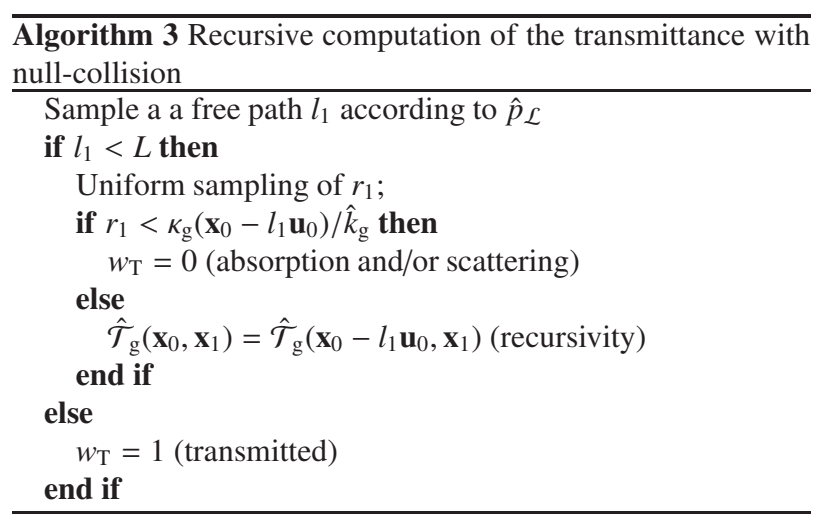

\section{Decomposition of the intensity}

\subsection{Principle}

The procedure presented so far makes it possible to construct a path, which allows the RTE to be solved within a heterogeneous semi-transparent medium. The construction of one radiative path (one realization of the algorithm) will stop when a boundary condition is reached (no emission of the medium). By considering the problem of a source partially illuminating the scene, we can highlight, thanks to the algorithmic interpretation, a convergence problem. Indeed, the only way to link the detector to the source and obtain a non-zero weight for the algorithm would be to undergo a scattering event within the laser layer in the exact direction of propagation of the laser source. However, the probability that the sampled direction of scattering is exactly equal to the direction of propagation of the source is zero. As it stands, all the realizations of the MC algorithm would bring zero weight and absolutely no convergence would be possible.

In order to overcome this problem, the main idea is to separate the intensity into two contributions such that:

$$
I\left(\mathbf{x}_{j}, \mathbf{u}_{j}\right)=I^{\mathrm{dir}}\left(\mathbf{x}_{j}, \mathbf{u}_{j}\right)+I^{\text {scat }}\left(\mathbf{x}_{j}, \mathbf{u}_{j}\right)
$$

where $I^{\mathrm{dir}}$ is the direct intensity or, in other words, the part of the energy directly transmitted by the source at the position $\mathbf{x}_{j}$ and in the direction $\mathbf{u}_{j}$. The second part of the right-hand side $I^{\text {scat }}$ corresponds to the rest of the energy, which is not due to direct transmission. Hence, the latter is due to, at least, one scattering event before reaching the position $\mathbf{x}_{j}$ in the direction $\mathbf{u}_{j}$ (in-scattered intensity). Very recent work applied such decomposition, called the local estimate technique, to solve radiative transfers in a highly resolved cloudy atmosphere [10].

The main difference between an atmospheric problem and the problem considered here lies in the aspect of partial illumination of the scene. Indeed, in the first case, the direct intensity is zero unless $-\mathbf{u}_{j} \in \Omega^{*}$ with $\Omega^{*}$ the solid angle of the source at the position $\mathbf{x}_{j}$. In our case, the direct intensity is zero unless $-\mathbf{u}_{j} \in \Omega^{*}$ and $\mathbf{x}_{j} \in \mathcal{N}$ with $\mathcal{N}$ the part of the volume $\mathcal{V}$ occupied by the laser sheet.

By injecting eq. (16) in eq. (3), the intensity is written as three components:

$$
\begin{aligned}
& I\left(\mathbf{x}_{0}, \mathbf{u}_{0}\right)=I^{\mathrm{dir}}\left(\mathbf{x}_{0}, \mathbf{u}_{0}\right) \\
& +\int_{0}^{\infty} \mathrm{d} \sigma_{1} \kappa_{\mathrm{s}}\left(\mathbf{x}_{1}\right) \mathcal{T}_{\mathrm{a}+\mathrm{s}}\left(\mathbf{x}_{0}, \mathbf{x}_{1}\right) \int_{4 \pi} \mathrm{d} \mathbf{u}_{1} \Phi\left(\mathbf{x}_{1}, \mathbf{u}_{0} \mid \mathbf{u}_{1}\right) I^{\mathrm{dir}}\left(\mathbf{x}_{1}, \mathbf{u}_{1}\right) \\
& +\int_{0}^{\infty} \mathrm{d} \sigma_{1} \kappa_{\mathrm{s}}\left(\mathbf{x}_{1}\right) \mathcal{T}_{\mathrm{a}+\mathrm{s}}\left(\mathbf{x}_{0}, \mathbf{x}_{1}\right) \int_{4 \pi} \mathrm{d} \mathbf{u}_{1} \Phi\left(\mathbf{x}_{1}, \mathbf{u}_{0} \mid \mathbf{u}_{1}\right) I^{\text {scat }}\left(\mathbf{x}_{1}, \mathbf{u}_{1}\right)
\end{aligned}
$$

with:

$$
I^{\mathrm{dir}}\left(\mathbf{x}_{j}, \mathbf{u}_{j}\right)=I^{*} \mathcal{T}_{\mathrm{a}+\mathrm{s}}\left(\mathbf{x}^{*}, \mathbf{x}_{j}\right) \mathcal{H}\left(-\mathbf{u}_{j} \in \Omega^{*}\right) \mathcal{H}\left(\mathbf{x}_{j} \in \mathcal{N}\right)
$$

where $I^{\mathrm{dir}}$ is the intensity emitted by the laser source $I^{*}$ and attenuated by absorption and scattering $\mathcal{T}_{\mathrm{a}+\mathrm{s}}$ between the source position $\mathbf{x}^{*}$ and the position $\mathbf{x}_{j}$. The three components in eq. (17) can be interpreted as:

$$
\begin{aligned}
I\left(\mathbf{x}_{0}, \mathbf{u}_{0}\right)= & \underbrace{I^{\mathrm{dir}}\left(\mathbf{x}_{0}, \mathbf{u}_{0}\right)}_{=0 \text { unless }\left(\mathbf{x}_{0} \in \mathcal{N}\right) \cap\left(\mathbf{u}_{0} \in \Omega^{*}\right)}+\underbrace{I^{\mathrm{s} 1}\left(\mathbf{x}_{0}, \mathbf{u}_{0}\right)}_{\text {higher order of scattering (recursivity) }} \\
& +\underbrace{I^{\mathrm{s} 2}\left(\mathbf{x}_{0}, \mathbf{u}_{0}\right)}_{\text {unless one scattering in } \mathcal{N}}
\end{aligned}
$$

In the latter equation (cf.eq. (19)), the direct intensity at the initialisation is non-zero unless the first position is inside the 


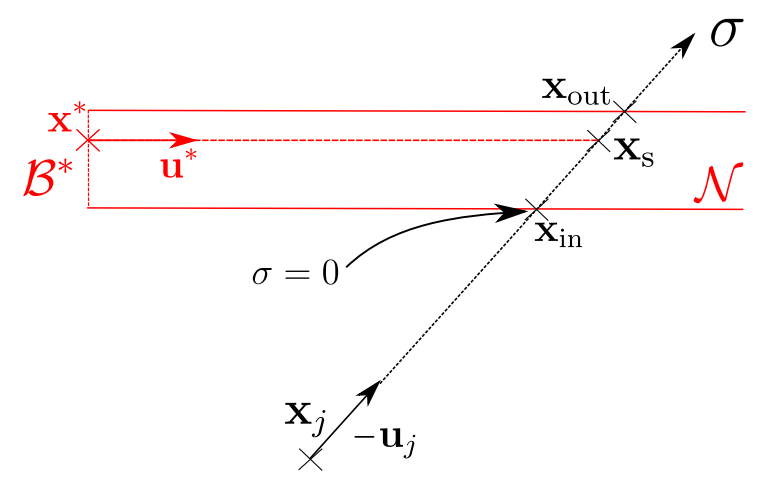

Figure 3: Part of the intensity transmitted by the source at the position $\mathbf{x}_{j}$ and in the direction $\mathbf{u}_{j}$ : computing $I^{\mathrm{s} 1}$ (cutting plane)

illuminated volume and if the first direction is inside the solid angle of the source. The second term $I^{\text {s1 }}$ represents the part of the intensity emitted by the source, directly transmitted to the position $\mathbf{x}_{1}$, scattered in the direction considered $\mathbf{u}_{0}$ and reaches the considered position $\mathbf{x}_{0}$. Thus, this term is zero if there is no intersection between $-\mathbf{u}_{0}$ starting from $\mathbf{x}_{0}$ and the laser sheet. Finally, the term $I^{\mathrm{s} 2}$ represents the part of the intensity emitted by the source but reaching the position $\mathbf{x}_{0}$ in the direction $\mathbf{u}_{0}$ with more than one scattering event. Hence, such a term is computed recursively, as presented in section 2.2 and section 2.4.

The direct intensity appears only at the initialisation. For $j>0$, only at-least-once-scattered intensity is computed. Thus, eq. (19) can be expressed with the following recursive form:

$$
I^{\mathrm{scat}}\left(\mathbf{x}_{j}, \mathbf{u}_{j}\right)=\underbrace{I^{\mathrm{s} 1}\left(\mathbf{x}_{j}, \mathbf{u}_{j}\right)}_{=0 \text { unless scattering in } \mathcal{N} \text { at order } \mathrm{j}+1}+\underbrace{I^{\mathrm{s} 2}\left(\mathbf{x}_{j}, \mathbf{u}_{j}\right)}_{\text {order }>j+1 \text { (recursivity) }}
$$

The following section concerns the algorithmic interpretation of the once-scattered intensity $I^{\text {s1 }}$.

\subsection{Computation of the once-scattered intensity}

The second term of the right-hand side of the eq. (17) is nonzero for positions $\mathbf{x}_{1}$ inside the laser sheet $\mathcal{N}$. The recursive form of the once-scattered intensity is then expressed as:

$$
\begin{aligned}
I^{\mathrm{s} 1}\left(\mathbf{x}_{j}, \mathbf{u}_{j}\right) & =\int_{x_{\text {in }}}^{x_{\text {out }}} \mathrm{d} \sigma_{1} \kappa_{\mathrm{s}}\left(\mathbf{x}_{1}\right) \mathcal{T}_{\mathrm{a}+\mathrm{s}}\left(\mathbf{x}_{j}, \mathbf{x}_{1}\right) \\
& \times\left[\int_{4 \pi} \mathrm{d} \mathbf{u}_{1} \Phi\left(\mathbf{x}_{1}, \mathbf{u}_{j} \mid \mathbf{u}_{1}\right) I^{*} \mathcal{T}_{\mathrm{a}+\mathrm{s}}\left(\mathbf{x}^{*}, \mathbf{x}_{1}\right) \mathcal{H}\left(-\mathbf{u}_{1} \in \Omega^{*}\right)\right]
\end{aligned}
$$

As shown in fig. 3, $\mathbf{x}_{\text {in }}$ and $\mathbf{x}_{\text {out }}$ denote the positions where the $j^{\text {th }}$ segment enters and leaves the laser sheet along the direction $\mathbf{u}_{j}$, respectively. For the sake of clarity, we note $\mathbf{x}_{\mathrm{s}}$ all the positions inside the laser sheet $\mathcal{N}$.

Only the directions included inside the source angle solid $\Omega^{*}$ allow non-zero contributions. Thus, the latter equation is sim- plified as:

$$
\begin{aligned}
I^{\mathrm{s} 1}\left(\mathbf{x}_{j}, \mathbf{u}_{j}\right) & \approx \int_{x_{\text {in }}}^{x_{\text {out }}} \mathrm{d} \sigma_{1} \kappa_{\mathrm{s}}\left(\mathbf{x}_{\mathrm{s}}\right) \mathcal{T}_{\mathrm{a}+\mathrm{s}}\left(\mathbf{x}_{j}, \mathbf{x}_{\mathrm{s}}\right) \\
& \times\left[\Phi\left(\mathbf{x}_{\mathrm{s}}, \mathbf{u}_{j} \mid \mathbf{u}^{*}\right) I^{*} \mathcal{T}_{\mathrm{a}+\mathrm{s}}\left(\mathbf{x}^{*}, \mathbf{x}_{\mathrm{s}}\right) \Delta \Omega^{*}\right]
\end{aligned}
$$

where $\Delta \Omega^{*}$ is the solid angle of dispersion of the laser source. Finally, the part of the energy emitted by the laser source and which reaches the position $\mathbf{x}_{j}$ in the direction $\mathbf{u}_{j}$ can be expressed as:

$$
\begin{aligned}
I^{\mathrm{s} 1}\left(\mathbf{x}_{j}, \mathbf{u}_{j}, \mathbf{x}_{\mathrm{in}}\right) & =\mathcal{T}_{\mathrm{a}+\mathrm{s}}\left(\mathbf{x}_{j}, \mathbf{x}_{\mathrm{in}}\right) \int_{0}^{d} \mathrm{~d} \sigma_{1} \kappa_{\mathrm{s}}\left(\mathbf{x}_{\mathrm{s}}\right) \mathcal{T}_{\mathrm{a}+\mathrm{s}}\left(\mathbf{x}_{\mathrm{in}}, \mathbf{x}_{\mathrm{s}}\right) \\
& \times \Phi\left(\mathbf{x}_{\mathrm{s}}, \mathbf{u}_{j} \mid \mathbf{u}^{*}\right) \mathcal{T}_{\mathrm{a}+\mathrm{s}}\left(\mathbf{x}_{\mathrm{s}}, \mathbf{x}^{*}\right) I^{*} \Delta \Omega^{*}
\end{aligned}
$$

using $\mathcal{T}_{\mathrm{a}+\mathrm{s}}\left(\mathbf{x}_{j}, \mathbf{x}_{\mathrm{s}}\right)=\mathcal{T}_{\mathrm{a}+\mathrm{s}}\left(\mathbf{x}_{j}, \mathbf{x}_{\mathrm{in}}\right) \mathcal{T}_{\mathrm{a}+\mathrm{s}}\left(\mathbf{x}_{\mathrm{in}}, \mathbf{x}_{\mathrm{s}}\right)$. The total distance travelled throughout the laser sheet is $d=\left\|\mathbf{x}_{\text {out }}-\mathbf{x}_{\text {in }}\right\|$. Let us note that the product $I^{*} \Delta \Omega^{*}$ corresponds to the flux density per unit area of the laser $\varphi^{*}$. This physical characteristic of the laser emission is the ratio of the power emitted to the surface of emission at a given frequency.

In order to bring up a probability of a mean free path by scattering, the transmittance by extinction is separated such that $\mathcal{T}_{\mathrm{a}+\mathrm{s}}\left(\mathbf{x}_{\text {in }} \mid \mathbf{x}_{\mathrm{s}}\right)=\mathcal{T}_{\mathrm{s}}\left(\mathbf{x}_{\mathrm{in}} \mid \mathbf{x}_{\mathrm{s}}\right) \mathcal{T}_{\mathrm{a}}\left(\mathbf{x}_{\text {in }} \mid \mathbf{x}_{\mathrm{s}}\right)$. Thus, eq. (23) can be rewritten as:

$$
\begin{aligned}
I^{\mathrm{s} 1}\left(\mathbf{x}_{j}, \mathbf{u}_{j}\right) & =\int_{0}^{\infty} \mathrm{d} \sigma_{1} p_{\Sigma}\left(\sigma_{1}\right) \mathcal{H}\left(\sigma_{1}<d\right) \mathcal{T}_{\mathrm{a}}\left(\mathbf{x}_{\mathrm{in}}, \mathbf{x}_{\mathrm{s}}\right) \\
& \times \mathcal{T}_{\mathrm{a}+\mathrm{s}}\left(\mathbf{x}_{j}, \mathbf{x}_{\mathrm{in}}\right) \Phi\left(\mathbf{x}_{\mathrm{s}}, \mathbf{u}_{j} \mid \mathbf{u}^{*}\right) \mathcal{T}_{\mathrm{a}+\mathrm{s}}\left(\mathbf{x}_{\mathrm{s}}, \mathbf{x}^{*}\right) \varphi^{*}
\end{aligned}
$$

Let us note that the term $\int_{0}^{\infty} \mathrm{d} \sigma_{1} p_{\Sigma}\left(\sigma_{1}\right) \mathcal{H}\left(\sigma_{1}<d\right)$ corresponds to the absorptance by scattering between the positions $\mathbf{x}_{\text {in }}$ and $\mathbf{x}_{\text {out }}$ along the direction $\mathbf{u}_{j}$. This quantity is the complementary probability of a transmittance and is computed by sampling distance before scattering according to $p_{\Sigma}\left(\sigma_{1}\right)$ and verifying if the distance travelled is smaller or greater than the distance $d$. The MC weight is unitary if $\sigma_{1}<d$ and zero otherwise.

A uniform scattering coefficient $\hat{k}_{\mathrm{s}}$ is used (cf. section 2.4) and the null-collision method is applied:

$$
\begin{aligned}
& I^{\mathrm{s} 1}\left(\mathbf{x}_{j}, \mathbf{u}_{j}\right)=\int_{0}^{\infty} \mathrm{d} \sigma_{1} \hat{p}_{\Sigma}\left(\sigma_{1}\right) \\
& \times\left\{\begin{array}{l}
\mathcal{H}\left(\sigma_{1}>d\right) \times\{0\} \\
+\mathcal{H}\left(\sigma_{1}<d\right) \times\left\{\begin{array}{l}
\mathcal{P}_{\mathrm{s}}\left(\mathbf{x}_{\mathrm{in}}-\sigma_{1} \mathbf{u}_{j}\right)\left\{w_{I}^{\mathrm{s} 1}\right. \\
\mathcal{P}_{\mathrm{n}}\left(\mathbf{x}_{\mathrm{in}}-\sigma_{1} \mathbf{u}_{j}\right)\left\{I^{\mathrm{s} 1}\left(\mathbf{x}_{j}, \mathbf{u}_{j}, \mathbf{x}_{\mathrm{in}}-\sigma_{1} \mathbf{u}_{j}\right)\right\}
\end{array}\right.
\end{array}\right.
\end{aligned}
$$

where $w_{I}^{\mathrm{s} 1}=\hat{\mathcal{T}}_{\mathrm{a}+\mathrm{s}}\left(\mathbf{x}_{j}, \mathbf{x}_{\mathrm{in}}\right) \hat{\mathcal{T}}_{\mathrm{a}}\left(\mathbf{x}_{\mathrm{in}}, \mathbf{x}_{\mathrm{s}}\right) \hat{\mathcal{T}}_{\mathrm{a}+\mathrm{s}}\left(\mathbf{x}_{\mathrm{s}}, \mathbf{x}^{*}\right) \Phi\left(\mathbf{x}_{\mathrm{s}}, \mathbf{u}_{j} \mid \mathbf{u}^{*}\right) \varphi^{*}$ is the MC weight if $\sigma_{1}<d$ and if the collision is real. Moreover, a uniform extinction coefficient $\hat{k}_{\mathrm{a}+\mathrm{s}}=\hat{k}_{\mathrm{a}}+\hat{k}_{\mathrm{s}}$ is needed to compute each transmittance by extinction $\hat{\mathcal{T}}_{\mathrm{a}+\mathrm{s}}$.

In the particular case of $\mathbf{x}_{j} \in \mathcal{N}$, the once-scattered intensity can be computed simply by setting $\mathbf{x}_{\text {in }}=\mathbf{x}_{j}$. The transmittance $\hat{\mathcal{T}}_{\mathrm{a}+\mathrm{s}}\left(\mathbf{x}_{j}, \mathbf{x}_{\mathrm{in}}\right)$ would then be unitary. 


\subsection{Double Randomization for transmittances}

As highlighted above, computation of $I^{\mathrm{s} 1}\left(\mathbf{x}_{j}, \mathbf{u}_{j}\right)$ is based on the evaluation of three transmittances and one absorptance. As described in section 2.5, each of these quantities is also obtained by the use of a MC method and would therefore require the use of a large number of realizations. In order to avoid an increase in the number of total realizations to be carried out as well as a propagation of error, a strategy of double randomization is used [17]. The Beer's non-linearity being bypassed thanks to the use of null-collision, the latter strategy allows the integral quantity $I^{\mathrm{sl}}\left(\mathbf{x}_{j}, \mathbf{u}_{j}\right)$ to be expressed as a unique expectation. Thus, a single realization of the algorithm can be carried out for the calculation of each term of transmittance or absorptance. We note $w_{\mathrm{T} 2}, w_{\mathrm{T} 3}$ and $w_{\mathrm{T} 4}$ as one realization of the MC algorithm associated with the calculation of $\mathcal{T}_{\mathrm{a}+\mathrm{s}}\left(\mathbf{x}_{j} \mid \mathbf{x}_{\mathrm{in}}\right), \mathcal{T}_{\mathrm{a}}\left(\mathbf{x}_{\mathrm{in}} \mid \mathbf{x}_{\mathrm{s}}\right)$ and $\mathcal{T}_{\text {a+s }}\left(\mathbf{x}_{\mathrm{s}} \mid \mathbf{x}^{*}\right)$, respectively.

\subsection{Preferential sampling for computation of the absorptance}

Preferential sampling, which consists in arbitrarily modifying a probability density, is an efficient way to reduce the variance of the estimated quantity. The possibilities for modifications being numerous, we only propose here a designed preferential sampling adapted to the main cause of the increasing of the variance: computation of the absorptance.

Indeed, in eq. (25), a distance travelled before scattering $\sigma_{1}$ is sampled according to $\hat{p}_{\Sigma}$. In the case of an optically thin medium inside the laser sheet, which may be due either to a low thickness of the laser sheet or to low values of scattering coefficients, the vast majority of scattering paths sampled would be greater than the distance travelled $d$. Thus, a vast majority of realizations would retain a MC weight of zero and the variance would increase. One possibility to avoid the latter constraint is to normalize the probability density $\hat{p}_{\Sigma}$ in the interval $[0 ; d]$. The new arbitrary probability density $\tilde{p}_{\Sigma}$ is written:

$$
\tilde{p}_{\Sigma}=\frac{\hat{p}_{\Sigma}}{1-\exp \left(-\hat{k}_{\mathrm{s}} d\right)}=\frac{\hat{p}_{\Sigma}}{\hat{\alpha}_{\mathrm{s}}}
$$

where $\hat{\alpha}_{\mathrm{s}}$ corresponds to an arbitrary uniform absorptance by scattering along the distance travelled $d$.

The scattering paths sampled according to $\tilde{p}_{\Sigma}$ can not be greater than $d$. The bias introduced by the modification of the probability density is then compensated by a modification of the MC weight. Thus, eq. (25) is modified as:

$$
\begin{aligned}
& I^{\mathrm{s} 1}\left(\mathbf{x}_{j}, \mathbf{u}_{j}, \mathbf{x}_{\mathrm{in}}\right)=\int_{0}^{\infty} \mathrm{d} \sigma_{1} \tilde{p}_{\Sigma}\left(\sigma_{1}\right) \\
& \times\left\{\begin{array}{l}
\mathcal{H}\left(\sigma_{1}>d\right) \times\{0\} \\
+\mathcal{H}\left(\sigma_{1}<d\right) \times\left\{\begin{array}{l}
\mathcal{P}_{\mathrm{s}}\left(\mathbf{x}_{\mathrm{in}}-\sigma_{1} \mathbf{u}_{j}\right)\left\{\hat{\alpha}_{\mathrm{s}} w_{I}^{\mathrm{s} 1}\right\} \\
\mathcal{P}_{\mathrm{n}}\left(\mathbf{x}_{\mathrm{in}}-\sigma_{1} \mathbf{u}_{j}\right)\left\{\hat{\alpha}_{\mathrm{s}} I^{\mathrm{s} 1}\left(\mathbf{x}_{j}, \mathbf{u}_{j}, \mathbf{x}_{\mathrm{in}}-\sigma_{1} \mathbf{u}_{j}\right)\right\}
\end{array}\right.
\end{array}\right.
\end{aligned}
$$

The resulting MC algorithm is detailed in algorithm 4.

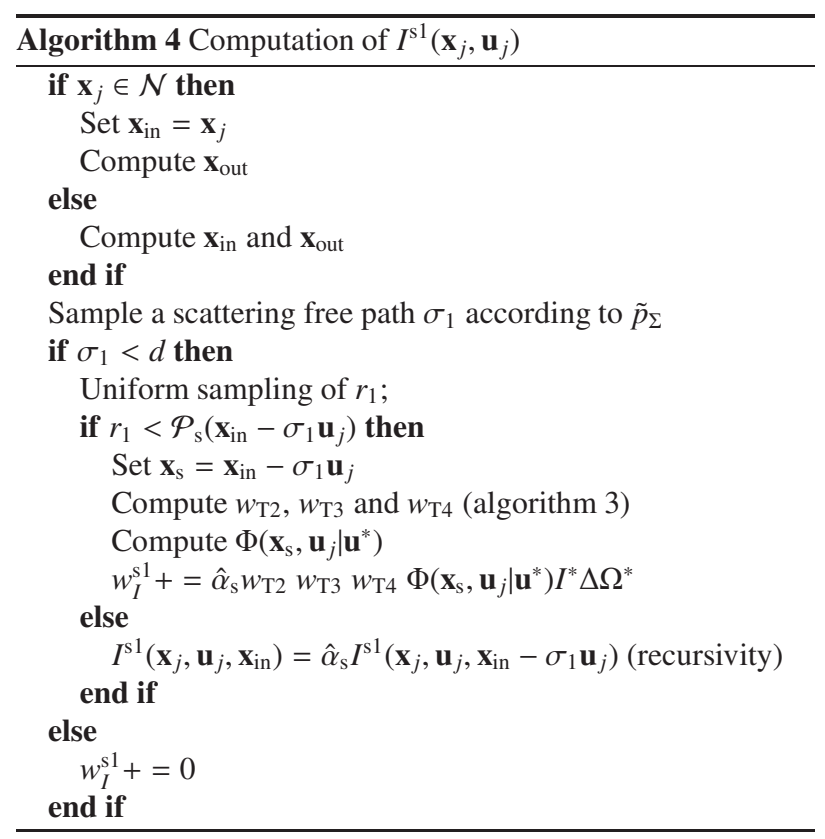

\section{Complete formulation of the algorithm}

The terms of direct intensity $I^{\mathrm{dir}}$ and once-scattered intensity $I^{\text {s1 }}$ having being made explicit, the last step is to express the term of higher order of scattering $I^{\mathrm{s} 2}$, which is computed recursively as explained in section 2.4. Using null-collision, the complete formulation allowing the resolution of the investigated problem can be expressed as:

$$
\begin{aligned}
I\left(\mathbf{x}_{0}, \mathbf{u}_{0}\right)= & I^{\mathrm{dir}}\left(\mathbf{x}_{0}, \mathbf{u}_{0}\right)+I^{\mathrm{s} 1}\left(\mathbf{x}_{0}, \mathbf{u}_{0}\right)+I^{\mathrm{s} 2}\left(\mathbf{x}_{0}, \mathbf{u}_{0}\right) \\
= & I^{\mathrm{dir}}\left(\mathbf{x}_{0}, \mathbf{u}_{0}\right)+I^{\mathrm{s} 1}\left(\mathbf{x}_{0}, \mathbf{u}_{0}\right)+\int_{0}^{\infty} \mathrm{d} \sigma_{1} \hat{p}_{\Sigma}\left(\sigma_{1}\right) \hat{\mathcal{T}}_{\mathrm{a}}\left(\mathbf{x}_{0}, \mathbf{x}_{1}\right) \\
& \times\left\{\begin{array}{l}
\mathcal{P}_{\mathrm{s}}\left(\mathbf{x}_{1}\right) \int_{4 \pi} \mathrm{d} \mathbf{u}_{1} \Phi\left(\mathbf{x}_{1}, \mathbf{u}_{0} \mid \mathbf{u}_{1}\right)\left(I^{\mathrm{s} 1}\left(\mathbf{x}_{1}, \mathbf{u}_{1}\right)+I^{\mathrm{s} 2}\left(\mathbf{x}_{1}, \mathbf{u}_{1}\right)\right) \\
+\left(1-\mathcal{P}_{\mathrm{s}}\right)\left(\mathbf{x}_{1}\right) \int_{4 \pi} \mathrm{d} \mathbf{u}_{1} \delta\left(\mathbf{x}_{1}, \mathbf{u}_{1}-\mathbf{u}_{0}\right) I^{\mathrm{s} 2}\left(\mathbf{x}_{1}, \mathbf{u}_{1}\right)
\end{array}\right.
\end{aligned}
$$

Let us note $w_{\mathrm{T} 1}$ as a realization of the MC algorithm associated with the calculation of $\mathcal{T}_{\mathrm{a}}\left(\mathbf{x}_{0}, \mathbf{x}_{1}\right)$. In the event of a fictitious collision (no change of direction $\mathbf{u}_{1}=\mathbf{u}_{0}$ ), with the order of the scattering events not increasing (rejection technique), the computation of the once-scattered intensity $I^{\mathrm{s} 1}\left(\mathbf{x}_{1}, \mathbf{u}_{1}\right)$ is not performed.

For $j>0$, the recursive form is:

$$
\begin{aligned}
& I^{\mathrm{scat}}\left(\mathbf{x}_{j}, \mathbf{u}_{j}\right)=I^{\mathrm{s} 1}\left(\mathbf{x}_{j}, \mathbf{u}_{j}\right)+I^{\mathrm{s} 2}\left(\mathbf{x}_{j}, \mathbf{u}_{j}\right) \\
& =I^{\mathrm{s} 1}\left(\mathbf{x}_{j}, \mathbf{u}_{j}\right)+\int_{0}^{\infty} \mathrm{d} \sigma_{j+1} \hat{p}_{\Sigma}\left(\sigma_{j+1}\right) \hat{\mathcal{T}}_{\mathrm{a}}\left(\mathbf{x}_{j}, \mathbf{x}_{j+1}\right) \\
& \times\left\{\begin{array}{l}
\mathcal{P}_{\mathrm{s}}\left(\mathbf{x}_{j+1}\right) \int_{4 \pi} \mathrm{d} \mathbf{u}_{1} \Phi\left(\mathbf{x}_{j+1}, \mathbf{u}_{j} \mid \mathbf{u}_{j+1}\right) I^{\mathrm{scat}}\left(\mathbf{x}_{j+1}, \mathbf{u}_{j+1}\right) \\
+\left(1-\mathcal{P}_{\mathrm{s}}\right)\left(\mathbf{x}_{j+1}\right) \int_{4 \pi} \mathrm{d} \mathbf{u}_{j+1} \delta\left(\mathbf{x}_{j+1}, \mathbf{u}_{j+1}-\mathbf{u}_{j}\right) I^{\mathrm{s} 2}\left(\mathbf{x}_{j+1}, \mathbf{u}_{j+1}\right)
\end{array}\right.
\end{aligned}
$$


Hence, three uniform radiative coefficients are needed:

- Absorbing $\hat{k}_{\mathrm{a}}$, useful for $\hat{\mathcal{T}}_{\mathrm{a}}$ computation

- Scattering $\hat{k}_{\mathrm{s}}$, useful for $\hat{p}_{\Sigma}$ and $\tilde{p}_{\Sigma}$ samplings

- Extinction $\hat{k}_{\mathrm{a}+\mathrm{s}}=\hat{k}_{\mathrm{a}}+\hat{k}_{\mathrm{s}}$, useful for $\hat{\mathcal{T}}_{\mathrm{a}+\mathrm{s}}$ computation

The complete resulting algorithm is fully described in fig. 4 . Interactions with walls are added.

- The starting point is to set the order of scattering $j$ to zero, to sample a direction $\mathbf{u}_{0}$ and a position $\mathbf{x}_{0}$ (step A1 and A2). Then, the direct intensity at the initialisation is computed (step A3) and weight of MC is initialized (step A4).

- The computation will loop on the backward and recursive MC branch (B2-B17) until an absorption event occurs in the medium or on the boundary. The quantity $\xi_{j}$ keeps track of the attenuation of the intensity by absorption in the medium all along the path composed by $j$ number of segments. If $\xi_{j+1}$ is 0 , the optical path is then interrupted and the Monte-Carlo weight of the $i^{\text {th }}$ realization is computed (step O1).

- More precisely, at each $j^{\text {th }}$ iteration, a scattering free path length is sampled according to $\hat{p}_{\Sigma}(\sigma)$ and a collision location is obtained (steps B2 and B3). A test on the distances traveled (B4) reveals whether the collision occurs in the medium or on the boundary. If it occurs in the medium, we follow the procedure associated with the infinite medium case (B4-B10) as described in algorithm 2. If it occurs on the boundary, a computation of the transmittance by absorption is first performed (step B12). Then, if no absorption event occurs (B13), a Bernoulli trial is used to identify if the path will be interrupted due to an absorption event (B15-O1) or will continue according to a reflection event (B15-B16).

- The implementation of the Monte-Carlo weight is performed through the source branch. For each new direction $\mathbf{u}_{j}$ sampled, a test is used to determine if an intersection between the laser sheet $\mathcal{N}$ and a ray starting from $\mathbf{x}_{j}$ in the direction $-\mathbf{u}_{j}$ exists (step B1). If an intersection $\mathbf{x}_{\text {in }}$ does not exist, the algorithm follows the main MonteCarlo branch presented previously. Otherwise, the algorithm follows the new specific source branch and computes the once-scattered intensity (S1-S10). The corresponding procedure is described in algorithm 4.

- The main idea is to iteratively determine if a scattering event occurs between the entry $\mathbf{x}_{\text {in }}$ and exit $\mathbf{x}_{\text {out }}$ location of the laser sheet (S2-S7). Index $k$ corresponds to the number of null-collision events. If no scattering event occurs and the path comes out of the laser sheet, the Monte-Carlo weight for the computation of the diffuse intensity is set to 0 (step S11).
- Otherwise, the weight is set to the part of energy which arises from the source and which is scattered at $\mathbf{x}_{\mathrm{S}}(\mathrm{S} 10-$ $\mathrm{S} 12)$. The output of the source branch joins the main branch at step B2.

The whole Monte-Carlo weight of a realization of this algorithm (with boundaries) is written as:

$$
w_{i}=I^{\mathrm{dir}}+\sum_{j=0}^{j_{\max }} \xi_{j} \mathcal{H}\left(\gamma_{j, s}\right) \hat{\alpha}_{\mathrm{s}}^{k_{\max }} w_{\mathrm{T} 2} w_{\mathrm{T} 3} w_{\mathrm{T} 4} \Phi\left(\mathbf{x}_{\mathrm{s}}, \mathbf{u}^{*} \mid \mathbf{u}_{j}\right) I^{*} \Delta \Omega^{*}
$$

where $j_{\max }$ is the index of the last collision before absorption by the medium or boundaries and $k_{\max }$ the index of the last fictitious collision before a scattering event occurs inside the laser sheet. $\mathcal{H}\left(\gamma_{j, s}\right)$ is equal to 1 if a new direction $\mathbf{u}_{j}$ is sampled (real collision), an intersection with $\mathcal{N}$ exists and a scattering event occurs inside the laser sheet. Hence, the estimation of $\bar{I}$ of $I\left(\mathbf{x}_{0}, \mathbf{u}_{0}\right)$ using $N$ realizations is given by:

$$
\bar{I}=\frac{1}{N} \sum_{i=1}^{N} w_{i}
$$

and the associated standard deviation is:

$$
\bar{\sigma}_{I}=\sqrt{\frac{1}{N(N-1)} \sum_{i=1}^{N}\left(w_{i}^{2}-\bar{I}^{2}\right)}
$$

\section{Results}

\subsection{Validation: Calculation of a flux}

\subsubsection{Studied geometry}

A parametric study in order to validate and evaluate the numerical behaviour of the above-described algorithm is now presented. The simple academic configuration as shown in fig. 1 is considered. The system is a cube, of side $L$ with cold purely absorbing faces, that are perpendicular to the $x, y$ and $z$ axes of a Cartesian coordinate system. The laser source is represented by a surface $S^{*}=L \times e$, which uniformly emits power $P_{\mathrm{e}}$ only in the direction $\mathbf{u}^{*}=\mathbf{y}$. A detector is assimilated to a cold and black absorbing surface of size $S_{\mathrm{a}}=l_{x} \times l_{y}$, centered in the middle of the bottom face and of normal $\mathbf{n}=\mathbf{z}$ perpendicular to the direction of propagation $\mathbf{u}^{*}$. A distance $D$ separates the lower part of the laser sheet from the detector. For the rest of the study, the geometrical parameters are set such that $e / L=0.005, l_{x} / L=l_{y} / L=0.25$ and $D / L=0.5$. Moreover, the Henyey-Greenstein single-scattering phase function $\Phi$ is used with a zero and uniform asymmetry parameter throughout the field.

\subsubsection{Validation procedure}

As seen previously, the work of integral formalism has allowed the construction of an optimized reverse algorithm that is intuitive with respect to path geometry and path construction. On the other hand, the construction of the weights in relation to the sampling choices (to control variance) are not immediate. 
A validation procedure is required. Thus, the proposed Optimized Reverse Monte-Carlo (ORMC) algorithm is compared to a well-mastered Analogous Monte-Carlo (AMC) algorithm. The total absorbed power $P_{\mathrm{a}}$ by the detector is computed with both methods. It is defined as:

$$
P_{\mathrm{a}}=\int_{S_{\mathrm{a}}} \mathrm{d} \mathbf{x}_{0} \int_{\left|\mathbf{u}_{0} \cdot \mathbf{n}\right|<0}^{2 \pi}\left|\mathbf{u}_{0} \cdot \mathbf{n}\right| I\left(\mathbf{x}_{0}, \mathbf{u}_{0}\right) \mathrm{d} \mathbf{u}_{0}
$$

On the one hand, in the case of the ORMC algorithm, paths start from the detector. In order to interpret the computation of the total absorbed power as a MC algorithm, eq. (33) is rewritten:

$$
P_{\mathrm{a}}=\int_{S_{\mathrm{a}}} \mathrm{d} \mathbf{x}_{0} p_{S}\left(\mathbf{x}_{0}\right) \int_{\left|\mathbf{u}_{0} \cdot \mathbf{n}\right|<0}^{2 \pi} \mathrm{d} \mathbf{u}_{0} p_{\Omega}\left(\mathbf{u}_{0}\right)\left\{S_{\mathrm{a}} \pi I\left(\mathbf{x}_{0}, \mathbf{u}_{0}\right)\right\}
$$

where $p_{S}\left(\mathbf{x}_{0}\right)=1 / S_{\mathrm{a}}$ and $p_{\Omega}\left(\mathbf{u}_{0}\right)=\left|\mathbf{u}_{0} \cdot \mathbf{n}\right| / \pi$ are the two probability densities associated with the sampling of a position on the absorbing surface and a direction, respectively. Both probability densities are chosen here such that the sampling is uniform. Computation of the incident intensity $I\left(\mathbf{x}_{0}, \mathbf{u}_{0}\right)$ is performed as described above with a reverse procedure. Hence, one realization of the algorithm consists in sampling the initial position $\mathbf{x}_{0}$ and a direction $\mathbf{u}_{0}$ (step A2 cf. fig. 4) and estimating the associated MC weight $w_{i}$ (cf. eq. (28)) then multiplying the latter by a factor $S_{\mathrm{a}} \pi$

On the other hand, in the case of the AMC algorithm, paths start from the source. One realization of the direct algorithm simply consists in sampling a position on the emitting surface $S^{*}$ in the direction $\mathbf{u}^{*}$ and building a radiative path based on absorption, scattering and null-collision events (cf. algorithm 2). The MC weight of the $i^{\text {th }}$ realisation is $w_{i}=P_{\mathrm{e}}$ if the path is interrupted on the detector and is zero otherwise.

In what follows, in order to consider the different steps of the validation chain while providing benchmark results for further improvement, three cases are investigated:

- Homogeneous medium solved as homogeneous

- Homogeneous medium solved as heterogeneous

- Heterogeneous medium

First of all, the medium is described by a set of uniform radiative properties and the resolution of the problem, with AMC and ORMC algorithms, is carried out as such. Thus, all the different exponential terms are computed analytically. This simple case allows us to validate the structure of the algorithm and, more specifically, the physical formulation of the diffuse intensity $I^{\mathrm{s} 1}$. Thereafter, the medium is still homogeneous but the computation is performed with the complete MC algorithm as described above (cf. fig. 4). Consequently, the probabilistic estimation of the different transmittances as well as the use of the principle of double randomization are verified. Finally, the case of a heterogeneous medium defined with analytical profiles is considered. The total procedure proposed, with the use of null-collision algorithms, is then validated.
Let us recall that the purpose of this comparison is not to identify which of AMC or ORMC is the most efficient in terms of computing time and convergence. The size of the detector is deliberately chosen to be large enough to allow the convergence of the AMC algorithm. The proposed benchmark, as well as the AMC, are presented solely for the purpose of validating the reverse formulation proposed. Thereafter, this generic algorithm could be generalized to the calculation of an intensity at a given point and in a given direction for all three-dimensional complex scenes. This computation-probe aspect of the ORMC will allow easy implementation and deal conveniently with the space and directional aspects (restricted) of the detector (pixels) when building computer-generated images.

\subsubsection{Homogeneous medium solved as homogeneous}

The radiative properties $\kappa_{\mathrm{a}}$ and $\kappa_{\mathrm{S}}$ being uniform, all the transmittances $\mathcal{T}$ and diffuse intensity $I^{\mathrm{s} 1}$ can be expressed and computed analytically. Hence, a null-collision algorithm is not used and a simplified version of the above-described procedure is implemented (not presented for the sake of brevity).

Table 1 displays the simulated values of the estimated total power absorbed $\bar{P}_{\mathrm{a}}$, the standard deviation and the relative standard deviation for several values of the optical thicknesses $\kappa_{\mathrm{a}} L$ and $\kappa_{\mathrm{S}} L$ obtained with the AMC and ORMC algorithms using $10^{6}$ independent realizations. The columns labelled $|\varepsilon|$ correspond to the absolute difference on the value estimated and $\dot{\sigma}$ the ratio of the AMC standard deviation to the ORMC standard deviation, respectively.

Firstly, one can note that the total power absorbed is very low compared to the total power emitted. The ratio $\bar{P}_{\mathrm{a}} / P_{\mathrm{e}}$ does not exceed a value of $0.44 \%$ for the chosen set of parameters. Thus, a very small amount of the emitted energy reaches the detector. This highlights the complexity in terms of convergence of the problem to be solved. However, the relative uncertainty $\bar{\sigma} / P_{\mathrm{a}}$ attests to a good level of convergence (below $2 \%$ for AMC and $0.6 \%$ for ORMC in all cases). By comparing the difference $|\varepsilon|$ with the standard deviations, we can state that the total power absorbed $\bar{P}_{\text {a }}$ associated with AMC and ORMC are indeed statistically compatible for low and high optical thicknesses. Thus, the proposed methodology for the simple case of a homogeneous medium is validated. Let us note that, for a given number of realizations with ORMC, the relative variance increases with the increase in the optical thickness of the medium, whether by absorption and/or scattering. This is simply explained by the fact that it becomes more and more difficult to connect the detector to the source with the increase in the opacity of the medium. The number of positive contributions to the MC algorithm decreases with the increase of the extinction (absorption and/or scattering). In the case of an optically thick medium, methodological improvements of the MC algorithm could be brought [18].

\subsubsection{Homogeneous medium solved as heterogeneous}

In this part, the complete procedure as described in fig. 4 is now applied to the case of a homogeneous medium. Each trans- 
mittance is computed numerically using a specific Monte Carlo procedure (double randomization). For simplicity, the upperbounds $\hat{k}_{\mathrm{s}}, \hat{k}_{\mathrm{a}}$ and $\hat{k}_{\mathrm{a}+\mathrm{s}}$ are chosen to be uniform throughout the field and are set to values of $\kappa_{\mathrm{a}}, \kappa_{\mathrm{s}}$ and $\kappa_{\mathrm{a}}+\kappa_{\mathrm{s}}$, respectively. In other words, the probability to meet a null-collision is naturally set to zero and all collisions are thus real (scattering, absorption or extinction).

Table 2 displays the same quantities for the same set of parameters as in the previous case (cf. table 1) using $10^{7}$ independent realizations. For $\kappa_{\mathrm{a}} L \in[0.5 ; 2.0]$, relative uncertainties show a good level of convergence (below $1.5 \%$ for AMC and $0.7 \%$ for ORMC in all values of $\kappa_{\mathrm{s}} L$ investigated). For higher values of $\kappa_{\mathrm{a}} L$, we notice an intermediate level of convergence with relative uncertainties above $4.5 \%$ for AMC and $1.5 \%$ for ORMC. Considering the values of standard deviation $\bar{\sigma}$ and the difference on the estimated value $|\varepsilon|$, estimations performed with AMC and ORMC are statistically compatible and the procedure is validated.

Let us note that approximately the same order of magnitude on the uncertainty as the previous study is obtained for ten times more MC realizations. This point can be easily explained by the error arising from the stochastic estimation of the three transmittances and the absorptance. Hence, the uncertainty associated with AMC and ORMC increase the total uncertainty of the desired computed quantity. Finally, the same remark as previously (cf. section 5.1.3) regarding the link between the uncertainty and the optical thickness of the medium can be made.

For the chosen set of parameters, table 3 displays the computation times obtained with an "Intel i5 - 2.4 GHz" CPU without any parallelization. Let us note that the proposed algorithm, based on local estimate techniques, has no specificity in terms of parallelization, and is therefore naturally fitted to parallel computing frameworks [19]. In a first approximation, its computation time decreases linearly with the number of processors involved in the calculation. For each MC algorithm, computation time $t$ to perform $10^{7}$ independent realizations, computation time $t_{1 \%}$ to reach $1 \%$ statistical uncertainty and ratio $t_{1 \%}^{\mathrm{AMC}} / t_{1 \%}^{\mathrm{ORMC}}$ are provided. As remarked above (cf. fig. 1 c)), for a given number of realizations of the MC algorithm, the proposed optimized algorithm, based on a main path plus a sub-path for once-scattered intensity, is about twice as slow as the AMC based on a unidirectional (single) path. Nevertheless, the time needed to reach $1 \%$ statistical error is much lower for ORMC than for AMC thanks to the convergence gain produced by this subpath. We note that the proposed reverse algorithm is particularly efficient for optically thin medium. For $\kappa_{\mathrm{a}, \max } L=0.5 \kappa_{\mathrm{s}, \max } L=0.5$, AMC needs $13.66 \mathrm{~s}$ to reach $1 \%$ statistical uncertainty while only $0.39 \mathrm{~s}$ is sufficient for ORMC. On the one hand, the increase in absorption increases the difficulty of connecting the detector to the source. Thus, for a given scattering coefficient, the time $t$ is quite constant and the time $t_{1 \%}$ increases. On the other hand, the increase in scattering increases the number of scattering events to be performed and the statistical uncertainty. Consequently, an increase of the times $t$ and $t_{1 \%}$ is noticed.

\subsubsection{Heterogeneous medium}

The last validation step consists in applying the methodology to a heterogeneous medium. The latter is defined by a nonuniform radiative properties field which is given by an analytical profile:

$$
\begin{aligned}
& \kappa_{\mathrm{a}}(\mathbf{x})=\kappa_{\mathrm{a}, \max }\left(\frac{L / 2-x}{L}\right)\left(1-\sqrt{\frac{(y-L / 2)^{2}+(z-L / 2)^{2}}{L^{2} / 2}}\right) \\
& \kappa_{\mathrm{S}}(\mathbf{x})=\kappa_{\mathrm{s}, \max }\left(\frac{L / 2-x}{L}\right)\left(1-\sqrt{\frac{(y-L / 2)^{2}+(z-L / 2)^{2}}{L^{2} / 2}}\right)
\end{aligned}
$$

featuring an axisymmetric flame along the $\mathrm{x}$ axis (maximum radiative property along the axis centered on $y=L / 2$ and $z=L / 2$ and a linear decay as a function of the distance to the axis, down to zero at the corners).

As previously, the upper-bounds $\hat{k}_{\mathrm{a}}, \hat{k}_{\mathrm{s}}$ and $\hat{k}_{\mathrm{a}+\mathrm{s}}$ are chosen as uniform and are set to $\kappa_{\mathrm{a}, \max }, \kappa_{\mathrm{s}, \max }$ and $\kappa_{\mathrm{a}, \max }+\kappa_{\mathrm{s} \text {,max }}$, respectively. Table 4 displays the same quantities as previous studies (cf. table 1 and table 2) for several values of the maximum optical thicknesses $\kappa_{\mathrm{a}, \max } L$ and $\kappa_{\mathrm{s}, \max } L$ obtained with both the AMC and ORMC algorithm and using $10^{7}$ independent realizations. Table 5 displays the corresponding computation time. Figure 5 allows a graphic comparison of the total power absorbed as a function of the maximum scattering optical thickness for three different profiles of radiative field:

- the homogeneous medium (uniform profile)

- linear (linear evolution along the $\mathrm{x}, \mathrm{y}$ and $\mathrm{z}$ axes with maximum reached for coordinate $\mathbf{x}=(L / 2, L, L)$

- the axisymmetric flame (cf. eq. (35) and eq. (36))

Each simulation is performed for an unitary absorbing optical thickness and computed with both AMC and ORMC using $10^{7}$ independent realizations. Each value is represented with the associated uncertainty (three times the estimated standard deviation).

Regarding the uncertainties and the absolute difference $|\varepsilon|$ in table 4 or results represented in fig. 5, there is no doubt of the statistical compatibility between AMC and ORMC, or that the proposed reverse procedure (cf. fig. 4) allows an unbiased and precise estimation of the incident radiative flux on the detector. In fig. 5, the differences in the variation of the absorbed power as a function of the maximum scattering thickness come, obviously, from a difference in the distribution of the radiative field as well as from the average optical thickness of the medium under consideration. The same observations as previously stated (cf. section 5.1.3 and section 5.1.4) regarding the link between the uncertainty, the computation time, and the optical thickness of the medium can be made. Note that since the axisymmetric flame profile is, on average, optically thinner than the homogeneous profile case, the convergence and computation times, for a given set of $\kappa_{\mathrm{a} \text {, max }}$ and $\kappa_{\mathrm{s} \text {, max }}$, are lower. Results obtained on this academic case provide a benchmark solution and could 
therefore be used for further algorithmic improvements.

Figure 6 simultaneously addresses the issue of the preferential sampling performed (cf. section 3.4) and the influence of the choice of the uniform upper-bound $\hat{k}$ on the performances. The relative statistical uncertainty, the computation time $t$ for $10^{6}$ independent realizations and the computation time $t_{1 \%}$ for $1 \%$ statistical uncertainty are drawn for varying values of $\rho$ such as $\hat{k}_{\mathrm{s}}=\rho \kappa_{\mathrm{s} \text {,max }}$. Results obtained with AMC, ORMC without preferential sampling and ORMC with preferential sampling are represented. On the one hand, the interest of using preferential sampling is highlighted. Due to the small optical thickness of the laser sheet, it is essential to favour scattering event along the distance travelled through the laser sheet to increase non zero contributions and, thus, decrease the relative statistical error. Regarding fig. 6 c) and d), preferential sampling does not increase the computation time for a given number of realizations of the algorithm and allows a strong reduction of computation time necessary to reach $1 \%$ statistical uncertainty (cf. fig. 6 e) and $\mathrm{f})$ ).

On the other hand, the use of preferential sampling with NCA leads to a dependence between convergence of the algorithm and the choice of the upper bound. This can be explained simply by the expression of the preferential sampling performed (cf.eq. (26)). The upper-bound appears in the retained weight of Monte-Carlo. As represented in fig. 6 a) and b), the relative statistical uncertainty of ORMC can strongly increase with the increase of the upper-bound $\hat{k}$. Convergence of AMC and ORMC with no preferential sampling is insensitive to the choice of the upper-bound. This is in good agreement with the results of Galtier et al. [6] and Eymet et al. [7]. Therefore, an appropriate choice of upper bounds is especially necessary when using a MC algorithm based on null-collision and preferential sampling. The use of acceleration grids (naturally related to the practice of NCA) allows to meet this particular need [10].

\subsection{Application: Image rendering of inhomogeneous media}

The algorithm presented in this article allows the calculation of the intensity at a given position and in a given direction while freeing itself from the constraints due to particularities of the laser emission. Hence, the proposed methodology (cf. section 4) can be used in a wide number of applications in order to simulate the presence of such types of source, which partially illuminate the studied scene. As performed in the above section for validation purposes (cf. section 5.1), integration of the intensity makes it easy to perform the computation of radiative quantity such as the net flux exchanged (for walls) or the divergence of the radiative flux (for volumes). Moreover, by integrating the intensity over the solid angle of each pixel of a camera, such reverse algorithms can also be useful for building computer-generated images. They make it possible to obtain a visual rendering of the radiative transfer and to illustrate the implementation of radiative-transfer algorithms in inhomogeneous media. Moreover, images obtained experimentally can be compared to images produced numerically and, then, serve as useful support for analysis such as the verification of commonly-made assumptions. Future work will concern the study of the morphology of soot by studying such images.
The generic algorithm presented here corresponds to a first step to produce computer-generated images for such kinds of laserlike source, as is the case of sooting flames. An academic situation is treated in this paper for proof of concept and validation issues. This algorithm can be straightforwardly applied to treat real flames inside complex geometries described thanks to Large Eddy Simulation. Such properties are due to the use of computer graphics tools coupled with NCA that allow orthogonality between the algorithm and the data provided.

Benchmark images were carried out on the academic configuration presented in fig. 7. Purely absorbing walls $(\varepsilon=1)$ are considered. The semi-transparent medium is described by analytical profiles for absorption and scattering. The same three types of analytical profile as used above (cf. section 5.1) are investigated here also. These are given in fig. 7 . The bottom left corner of the laser is located at coordinate $(L / 2,0, L / 2)$. The thickness of the laser sheet $e$ is set such that $e / L=0.005$. The energy emitted propagates accordingly in the $\mathbf{x}$ direction. The camera is located at coordinate $(L, L / 2, L / 2)$ and perpendicularly to the laser propagation direction. The field of view (fov) of the camera is set to 55 .

Figure 8 represents the obtained images for each three profiles (lines), for three different scattering optical thicknesses (columns) such that $\kappa_{\mathrm{s}, \max } L=1, \kappa_{\mathrm{s}, \max } L=2$ and $\kappa_{\mathrm{s}, \max } L=5$. A unitary absorption optical thickness $\kappa_{\mathrm{a} \text {, } \max } L$ is set and 30000 realizations of the MC algorithm per pixel are performed. Images are built with $320 \times 180$ pixels. The considered problem being monochromatic, images are thus in greyscale. The obtained images give a good illustration of the scattering behaviour of each profile studied and appear as the radiative signature of the medium. Light scattering increases with the increase in the maximum optical thickness. Therefore, light is scattered further and further away from the laser sheet. For the homogeneous medium and $\kappa_{\mathrm{s}, \max } L=1$, all the laser sheet is visible. This corresponds mainly to the first order of scattering. Then, with the increase in the scattering, the maximum of intensity gets closer to the left part of the image, i.e the location of the laser emission. In the case of the linear profile, because the medium is scattering more and more linearly with the increase in the coordinates, the maximum intensity is reached on the right side of the image. Hence, one can see that light transport is greater on the upper part than on the lower part. Finally, in the case of the simili flame profile, the laser sheet is no longer visible on the left and on the right part due to the lack of scattering in this part of the domain. Then, the axisymmetric profile is visible and light transport is also logically more emphasized on the bottom part than on the upper part.

\section{Conclusion}

The study of the propagation of a laser by absorption and scattering in heterogeneous media can be complex due to the small directional and spatial extent of such an emitting source. The main difficulty lies in the ability to solve the convergence problems caused by a source that partially illuminates the studied scene. Usually, assumptions are made, such as a low order of scattering, or a homogeneous medium, or by using light field 
pre-calculations.

In order to bypass this difficulty, we developed a flexible and simply-implementable method based on a Backward (Reverse) Monte-Carlo algorithm. The convenient framework proposed by Galtier et al.[6] allows us to design, by means of the integral formulation and the null-collision algorithm, a specific and designed Monte-Carlo algorithm, which integrates the heterogeneity of the radiative property field and the constraints related to a laser-like source. Convergence constraints due to laser emission were avoided thanks to the separation of the intensity into direct/diffuse terms and an adapted working of the integral formulation. Such a decomposition made it possible to highlight the once-scattered intensity, which corresponds to the part of the energy reaching a given position in a given direction after only one scattering inside the laser sheet, which can be estimated with a specific Monte-Carlo Algorithm. Thus, MonteCarlo weight could be increased more continuously, even for scattering positions outside the laser sheet and thus, the standard deviation is decreased. The whole demonstration and the methodological procedure, from the integral formulation to the different Monte-Carlo algorithms required, were developed.

The presented method allows computation of the intensity at a given position and in a given direction (probe computation). Thus, on the one hand, divergence of the radiative flux (volumes) or absorbed flux (walls) can be estimated. A validation procedure was carried out by comparing the total absorbed flux with both the present approaches and a well-mastered analogous Monte-Carlo algorithm. Results showed, for the three cases investigated, a very good agreement and statistical compatibility for a wide range of absorbing and scattering optical thicknesses. Furthermore, such reverse algorithms (probe computation) are particularly well adapted for building computergenerated images. Hence,the implementation of this new radiative transfer algorithm allowed such images to be built efficiently in academic benchmark cases.

These new algorithmic developments and validations correspond to the first step in producing computer-generated images of combustion media. Thanks to the orthogonality between the algorithm and the data permitted by the NCA and the use of acceleration grids, future work can focus on obtaining a radiative property field from Large Eddy Simulation (LES) of sooting flames. This will allow the study of the influence of soot morphologies on the radiative transfer. Finally, because of current difficulties related to the reproduction of the exact conditions of an experimental physical measurement such as optical systems or the polarization state of light, an experimental confrontation is not feasible for the moment. However, this tool will provide a support function for the analysis of commonly made assumptions and experimental constraints such as trapping effects, the influence of species present and the impact of multiple scattering in soot characterization studies.

\section{Acknowledgements}

This work was supported by the ANR ASTORIA project, grant ANR-18-CE05-0015 of the French Agence Nationale de la Recherche. 


\begin{tabular}{lccccccccc}
\hline \multicolumn{3}{l}{ Optical thickness } & \multicolumn{3}{c}{ Analogous Monte Carlo } & \multicolumn{2}{c}{ Optimized Reverse Monte Carlo } & error & ratio \\
$\kappa_{\mathrm{a}} L$ & $\kappa_{\mathrm{s}} L$ & $\frac{\bar{P}_{\mathrm{a}}}{P_{\mathrm{e}}}(\%)$ & $\frac{\bar{\sigma}}{P_{\mathrm{e}}}(\%)$ & $\overline{\frac{\sigma}{\bar{P}_{\mathrm{a}}}}(\%)$ & $\frac{\bar{P}_{\mathrm{e}}}{P_{\mathrm{e}}}(\%)$ & $\frac{\bar{\sigma}}{P_{\mathrm{e}}}(\%)$ & $\frac{\bar{\sigma}}{\bar{P}_{\mathrm{a}}}(\%)$ & $|\varepsilon|(\%)$ & $\dot{\sigma}(-)$ \\
\hline 0.5 & 0.5 & 0.227595 & 0.003631 & 1.59 & 0.226742 & 0.000344 & 0.15 & 0.000850 & 10.6 \\
0.5 & 1.0 & 0.351265 & 0.004487 & 1.28 & 0.355024 & 0.000650 & 0.18 & 0.003761 & 6.9 \\
0.5 & 2.0 & 0.440055 & 0.004444 & 1.01 & 0.448159 & 0.001033 & 0.23 & 0.008100 & 4.3 \\
0.5 & 5.0 & 0.367875 & 0.004335 & 1.18 & 0.367485 & 0.001483 & 0.40 & 0.000410 & 2.9 \\
\hline 1.0 & 0.5 & 0.132405 & 0.002167 & 1.63 & 0.131976 & 0.000196 & 0.15 & 0.000431 & 11.1 \\
1.0 & 1.0 & 0.204412 & 0.003686 & 1.80 & 0.204405 & 0.000368 & 0.18 & 0.000013 & 10.0 \\
1.0 & 2.0 & 0.246245 & 0.002884 & 1.17 & 0.251915 & 0.000581 & 0.23 & 0.005669 & 5.0 \\
1.0 & 5.0 & 0.189424 & 0.002403 & 1.27 & 0.187337 & 0.000782 & 0.42 & 0.002069 & 3.1 \\
\hline 2.0 & 0.5 & 0.047177 & 0.000832 & 1.76 & 0.047128 & 0.000072 & 0.15 & 0.000052 & 11.5 \\
2.0 & 1.0 & 0.071320 & 0.001017 & 1.43 & 0.072042 & 0.000132 & 0.18 & 0.000719 & 7.7 \\
2.0 & 2.0 & 0.083775 & 0.001084 & 1.29 & 0.086263 & 0.000213 & 0.24 & 0.002485 & 5.3 \\
2.0 & 5.0 & 0.058105 & 0.000857 & 1.47 & 0.056526 & 0.000256 & 0.45 & 0.001582 & 3.3 \\
\hline 5.0 & 0.5 & 0.002814 & 0.000055 & 1.97 & 0.002834 & 0.000006 & 0.20 & 0.000013 & 10.0 \\
5.0 & 1.0 & 0.004204 & 0.000078 & 1.86 & 0.004268 & 0.000010 & 0.22 & 0.000058 & 8.3 \\
5.0 & 2.0 & 0.004725 & 0.000082 & 1.73 & 0.004932 & 0.000014 & 0.28 & 0.000197 & 6.0 \\
5.0 & 5.0 & 0.002861 & 0.000062 & 2.16 & 0.002681 & 0.000015 & 0.57 & 0.000180 & 4.1 \\
\hline
\end{tabular}

Table 1: Estimation, absolute and relative standard deviation as a function of optical thicknesses. Comparison of AMC and ORMC algorithm for $10^{6}$ independent realizations in the case of homogeneous medium (without NCA and double randomization technique) 


\begin{tabular}{|c|c|c|c|c|c|c|c|c|c|}
\hline \multicolumn{2}{|c|}{ Optical thickness } & \multicolumn{3}{|c|}{ Analogous Monte Carlo } & \multicolumn{3}{|c|}{ Optimized Reverse Monte Carlo } & \multirow{2}{*}{$\begin{array}{c}\text { error } \\
|\varepsilon|(\%)\end{array}$} & \multirow{2}{*}{$\begin{array}{l}\text { ratio } \\
\dot{\sigma}(-)\end{array}$} \\
\hline$\kappa_{\mathrm{a}, \max } L$ & $\kappa_{\mathrm{s}, \max } L$ & $\frac{\bar{P}_{\mathrm{a}}}{P_{\mathrm{e}}}(\%)$ & $\frac{\bar{\sigma}}{P_{\mathrm{e}}}(\%)$ & $\frac{\bar{\sigma}}{\bar{P}_{3}}(\%)$ & $\frac{\bar{P}_{\mathrm{a}}}{P_{\mathrm{e}}}(\%)$ & $\frac{\bar{\sigma}}{P_{\mathrm{e}}}(\%)$ & $\frac{\bar{\sigma}}{\bar{P}_{3}}(\%)$ & & \\
\hline 0.5 & 0.5 & 0.227439 & 0.001506 & 0.66 & 0.227139 & 0.000183 & 0.08 & 0.000300 & 8.2 \\
\hline 0.5 & 1.0 & 0.355959 & 0.001883 & 0.53 & 0.354822 & 0.000374 & 0.11 & 0.001137 & 5.0 \\
\hline 0.5 & 2.0 & 0.445549 & 0.002106 & 0.47 & 0.449592 & 0.000681 & 0.15 & 0.004042 & 3.1 \\
\hline 0.5 & 5.0 & 0.366999 & 0.001912 & 0.52 & 0.367606 & 0.001065 & 0.29 & 0.000606 & 1.8 \\
\hline 1.0 & $\overline{0.5}$ & 0.131239 & 0.001144 & 0.87 & 0.132406 & 0.000140 & 0.11 & 0.001166 & 8.1 \\
\hline 1.0 & 1.0 & 0.205819 & 0.001433 & 0.70 & 0.204397 & 0.000273 & 0.13 & 0.001422 & 5.2 \\
\hline 1.0 & 2.0 & 0.253779 & 0.001591 & 0.63 & 0.252198 & 0.000484 & 0.19 & 0.001581 & 3.3 \\
\hline 1.0 & 5.0 & 0.186849 & 0.001365 & 0.73 & 0.186661 & 0.000726 & 0.39 & 0.000188 & 1.9 \\
\hline 2.0 & 0.5 & 0.048280 & 0.000694 & 1.43 & 0.047170 & 0.000079 & 0.16 & 0.001109 & 8.8 \\
\hline 2.0 & 1.0 & 0.071990 & 0.000848 & 1.19 & 0.072006 & 0.000154 & 0.21 & 0.000016 & 5.5 \\
\hline 2.0 & 2.0 & 0.085860 & 0.000926 & 1.08 & 0.086795 & 0.000268 & 0.3 & 0.000935 & 3.4 \\
\hline 2.0 & 5.0 & 0.055720 & 0.000746 & 1.34 & 0.056183 & 0.000378 & 0.68 & 0.000463 & 2.0 \\
\hline 5.0 & 0.5 & 0.002770 & 0.000166 & 5.81 & 0.002840 & 0.000018 & 0.63 & 0.000070 & 9.2 \\
\hline 5.0 & 1.0 & 0.004190 & 0.000204 & 4.83 & 0.004256 & 0.000032 & 0.76 & 0.000067 & 6.3 \\
\hline 5.0 & 2.0 & 0.005010 & 0.000223 & 4.57 & 0.004906 & 0.000056 & 1.13 & 0.000103 & 4.0 \\
\hline 5.0 & 5.0 & 0.002640 & 0.000162 & 6.15 & 0.002736 & 0.000076 & 2.91 & 0.000097 & 2.0 \\
\hline
\end{tabular}

Table 2: Estimation, absolute and relative standard deviation as a function of optical thicknesses. Comparison of AMC and ORMC algorithm for $10^{7}$ independent realizations in the case of homogeneous medium (with NCA and double randomization technique)

\begin{tabular}{|c|c|c|c|c|c|c|}
\hline \multicolumn{2}{|c|}{ Optical thickness } & \multicolumn{2}{|c|}{ Analogous Monte Carlo } & \multicolumn{2}{|c|}{ Optimized Reverse Monte Carlo } & \multirow{2}{*}{$\begin{array}{c}\text { ratio } \\
\frac{t_{1 \% \%}^{\mathrm{AMC}}}{t_{1 \%}^{\text {ORMC }}}(-)\end{array}$} \\
\hline$\kappa_{\mathrm{a}, \max } L$ & $\kappa_{\mathrm{s}, \max } L$ & $t(\mathrm{~s})$ & $t_{1 \%}(\mathrm{~s})$ & $t(\mathrm{~s})$ & $t_{1 \%}(\mathrm{~s})$ & \\
\hline 0.5 & 0.5 & 31.84 & 13.66 & 63.30 & 0.39 & 34.67 \\
\hline 0.5 & 1.0 & 42.79 & 11.45 & 79.67 & 0.81 & 14.08 \\
\hline 0.5 & 2.0 & 66.18 & 14.03 & 115.46 & 2.51 & 5.57 \\
\hline 0.5 & 5.0 & 132.21 & 34.32 & 235.89 & 18.5 & 1.85 \\
\hline 1.0 & 0.5 & 31.74 & 22.85 & 63.21 & 0.76 & 29.92 \\
\hline 1.0 & 1.0 & 43.47 & 19.62 & 81.51 & 1.21 & 16.10 \\
\hline 1.0 & 2.0 & 65.23 & 24.32 & 113.5 & 4.32 & 5.62 \\
\hline 1.0 & 5.0 & 131.1 & 67.69 & 230.27 & 33.5 & 2.01 \\
\hline 2.0 & 0.5 & 31.55 & 66.86 & 62.32 & 1.47 & 45.38 \\
\hline 2.0 & 1.0 & 44.17 & 58.00 & 80.43 & 3.65 & 15.84 \\
\hline 2.0 & 2.0 & 65.55 & 73.43 & 115.8 & 9.90 & 7.41 \\
\hline 2.0 & 5.0 & 133.43 & 220.0 & 228.78 & 101.5 & 2.16 \\
\hline 5.0 & 0.5 & 32.46 & 1088.29 & 64.45 & 25.3 & 42.96 \\
\hline 5.0 & 1.0 & 44.08 & 1030.27 & 80.32 & 52.6 & 19.55 \\
\hline 5.0 & 2.0 & 69.12 & 1276.54 & 117.04 & 161.4 & 7.90 \\
\hline 5.0 & 5.0 & 130.4 & 4596.71 & 229.01 & 1820.2 & 2.52 \\
\hline
\end{tabular}

Table 3: Comparison of AMC and ORMC algorithm in the case of homogeneous medium (with NCA and double randomization technique); computation time $t$ for $10^{7}$ independent realizations, computation time $t_{1 \%}$ for $1 \%$ statistical uncertainty and ratio $t_{1}^{\mathrm{AMC}} / t_{1}^{\mathrm{ORMC}}$ between ORMC and AMC. This computation was performed with an "Intel i5 - $2.4 \mathrm{GHz}$ " CPU without any parallelization. 


\begin{tabular}{|c|c|c|c|c|c|c|c|c|c|}
\hline \multicolumn{2}{|c|}{ Optical thickness } & \multicolumn{3}{|c|}{ Analogous Monte Carlo } & \multicolumn{3}{|c|}{ Optimized Reverse Monte Carlo } & \multirow{2}{*}{$\begin{array}{c}\text { error } \\
|\varepsilon|(\%)\end{array}$} & \multirow{2}{*}{$\begin{array}{l}\text { ratio } \\
\dot{\sigma}(-)\end{array}$} \\
\hline$\kappa_{\mathrm{a}, \max } L$ & $\kappa_{\mathrm{s}, \max } L$ & $\frac{\bar{P}_{\mathrm{a}}}{P_{\mathrm{e}}}(\%)$ & $\frac{\bar{\sigma}}{P_{\mathrm{e}}}(\%)$ & $\frac{\bar{\sigma}}{\bar{P}_{3}}(\%)$ & $\frac{\bar{P}_{\mathrm{a}}}{P_{\mathrm{e}}}(\%)$ & $\frac{\bar{\sigma}}{P_{\mathrm{e}}}(\%)$ & $\frac{\bar{\sigma}}{\bar{P}_{3}}(\%)$ & & \\
\hline 0.5 & 0.5 & 0.129679 & 0.001138 & 0.88 & 0.131952 & 0.000119 & 0.09 & 0.002272 & 9.5 \\
\hline 0.5 & 1.0 & 0.237989 & 0.001540 & 0.65 & 0.240220 & 0.000251 & 0.10 & 0.002230 & 6.1 \\
\hline 0.5 & 2.0 & 0.404389 & 0.002007 & 0.50 & 0.401241 & 0.000512 & 0.12 & 0.003148 & 3.9 \\
\hline 0.5 & 5.0 & 0.630090 & 0.002502 & 0.40 & 0.632488 & 0.001135 & 0.18 & 0.002398 & 2.2 \\
\hline 1.0 & $\overline{0.5}$ & 0.107860 & 0.001037 & 0.96 & 0.109157 & 0.000109 & 0.10 & 0.001297 & 9.5 \\
\hline 1.0 & 1.0 & 0.196739 & 0.001401 & 0.71 & 0.198997 & 0.000225 & 0.11 & 0.002257 & 6.2 \\
\hline 1.0 & 2.0 & 0.335599 & 0.001828 & 0.54 & 0.333091 & 0.000455 & 0.14 & 0.002508 & 4.0 \\
\hline 1.0 & 5.0 & 0.526880 & 0.002289 & 0.43 & 0.525729 & 0.001009 & 0.19 & 0.001150 & 2.3 \\
\hline 2.0 & 0.5 & 0.075900 & 0.000870 & 1.14 & 0.075967 & 0.000091 & 0.12 & 0.000068 & 9.6 \\
\hline 2.0 & 1.0 & 0.139329 & 0.001179 & 0.84 & 0.138886 & 0.000184 & 0.13 & 0.000443 & 6.4 \\
\hline 2.0 & 2.0 & 0.233919 & 0.001527 & 0.65 & 0.234908 & 0.000373 & 0.16 & 0.000988 & 4.1 \\
\hline 2.0 & 5.0 & 0.373769 & 0.001929 & 0.51 & 0.373076 & 0.000827 & 0.22 & 0.000693 & 2.3 \\
\hline 5.0 & 0.5 & 0.030130 & 0.000548 & 1.82 & 0.029244 & 0.000054 & 0.19 & 0.000885 & 10.1 \\
\hline 5.0 & 1.0 & 0.054210 & 0.000736 & 1.35 & 0.054202 & 0.000112 & 0.21 & 0.000007 & 6.6 \\
\hline 5.0 & 2.0 & 0.092800 & 0.000962 & 1.04 & 0.093000 & 0.000225 & 0.24 & 0.000200 & 4.3 \\
\hline 5.0 & 5.0 & 0.152469 & 0.001233 & 0.81 & 0.151448 & 0.000491 & 0.32 & 0.001021 & 2.5 \\
\hline
\end{tabular}

Table 4: Estimation, absolute and relative standard deviation as a function of optical thicknesses. Comparison of AMC and ORMC algorithm for $10^{7}$ independent realizations in the case of heterogeneous medium: profile of an axisymmetric flame.

\begin{tabular}{|c|c|c|c|c|c|c|}
\hline \multicolumn{2}{|c|}{ Optical thickness } & \multicolumn{2}{|c|}{ Analogous Monte Carlo } & \multicolumn{2}{|c|}{ Optimized Reverse Monte Carlo } & \multirow{2}{*}{$\begin{array}{c}\text { ratio } \\
\frac{t_{1 \% \%}^{\mathrm{AMC}}}{t_{1 \%}^{\text {ORMC }}}(-)\end{array}$} \\
\hline$\kappa_{\mathrm{a}, \max } L$ & $\kappa_{\mathrm{s}, \max } L$ & $t(\mathrm{~s})$ & $t_{1 \%}(\mathrm{~s})$ & $t(\mathrm{~s})$ & $t_{1 \%}(\mathrm{~s})$ & \\
\hline 0.5 & 0.5 & 25.33 & 18.07 & 57.56 & 0.40 & 45.21 \\
\hline 0.5 & 1.0 & 30.56 & 12.21 & 67.36 & 0.95 & 12.80 \\
\hline 0.5 & 2.0 & 40.62 & 9.99 & 89.31 & 1.45 & 6.86 \\
\hline 0.5 & 5.0 & 71.82 & 11.05 & 152.83 & 4.76 & 2.32 \\
\hline 1.0 & 0.5 & 25.15 & 23.64 & 56.37 & 0.51 & 46.24 \\
\hline 1.0 & 1.0 & 30.27 & 15.13 & 66.68 & 0.82 & 18.34 \\
\hline 1.0 & 2.0 & 41.92 & 12.10 & 88.12 & 1.44 & 8.41 \\
\hline 1.0 & 5.0 & 74.02 & 13.56 & 145.61 & 5.32 & 2.54 \\
\hline 2.0 & 0.5 & 25.53 & 34.37 & 55.06 & 0.70 & 49.35 \\
\hline 2.0 & 1.0 & 31.96 & 22.61 & 67.39 & 1.09 & 20.81 \\
\hline 2.0 & 2.0 & 42.14 & 18.44 & 84.25 & 2.51 & 7.33 \\
\hline 2.0 & 5.0 & 72.48 & 19.85 & 151.66 & 6.89 & 2.88 \\
\hline 5.0 & 0.5 & 29.33 & 97.54 & 58.93 & 2.07 & 47.01 \\
\hline 5.0 & 1.0 & 35.14 & 63.69 & 69.22 & 3.25 & 19.59 \\
\hline 5.0 & 2.0 & 47.11 & 50.30 & 91.04 & 5.92 & 8.49 \\
\hline 5.0 & 5.0 & 77.18 & 51.86 & 157.03 & 17.11 & 3.03 \\
\hline
\end{tabular}

Table 5: Comparison of AMC and ORMC algorithm in the case of heterogeneous medium (axisymmetric flame); computation time $t$ for $10^{7}$ independent realizations, computation time $t_{1} \%$ for $1 \%$ statistical uncertainty and ratio $t_{1 \%}^{\mathrm{AMC}} / t_{1 \%}^{\mathrm{ORMC}}$ between ORMC and AMC. This computation was performed with an "Intel i5 - $2.4 \mathrm{GHz}$ " CPU without any parallelization. 


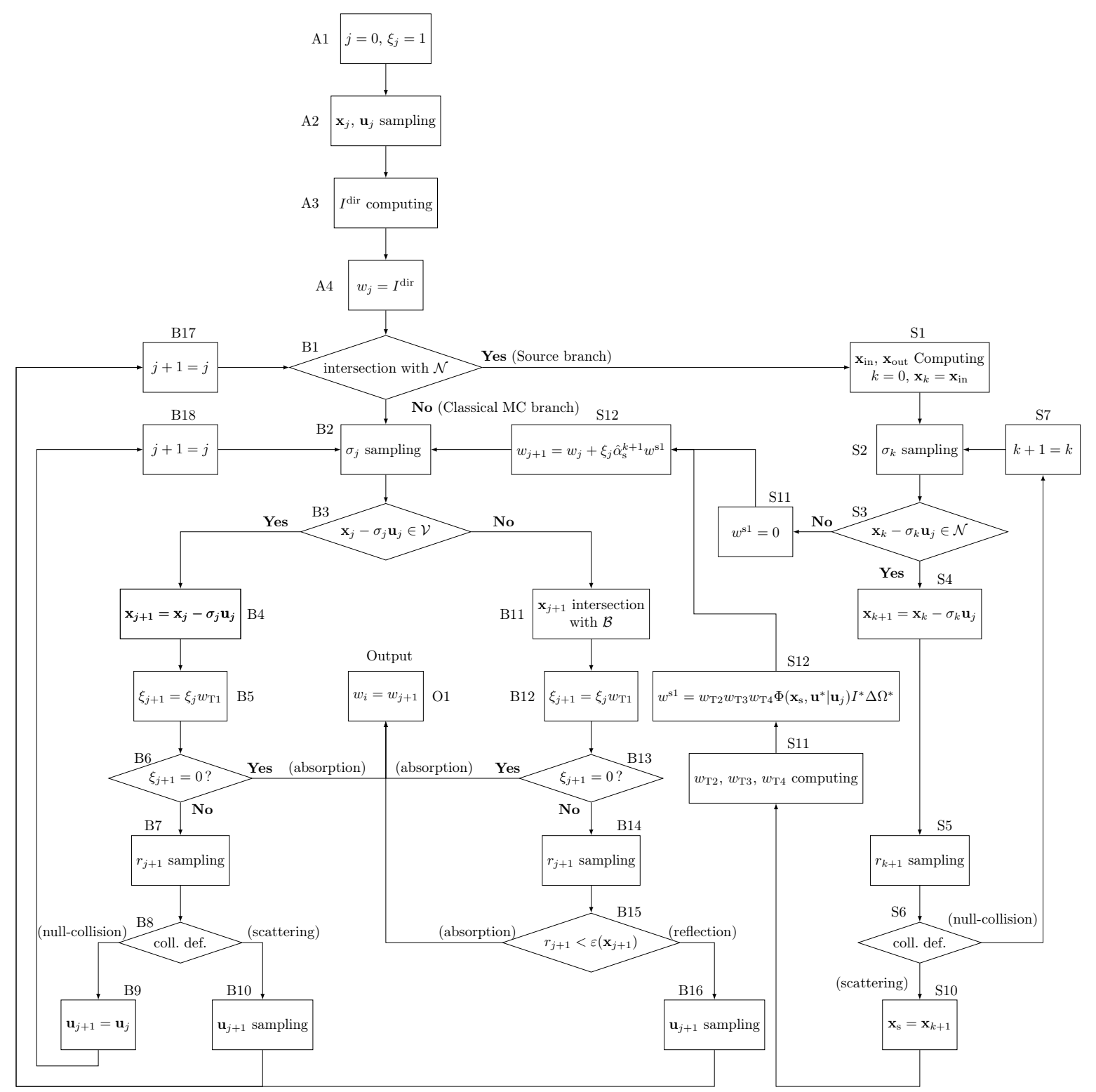

Figure 4: Description of the proposed algorithm. It follows a classical reverse Monte-Carlo with a specific branch associated with the integration of a source partially illuminating the scene. 


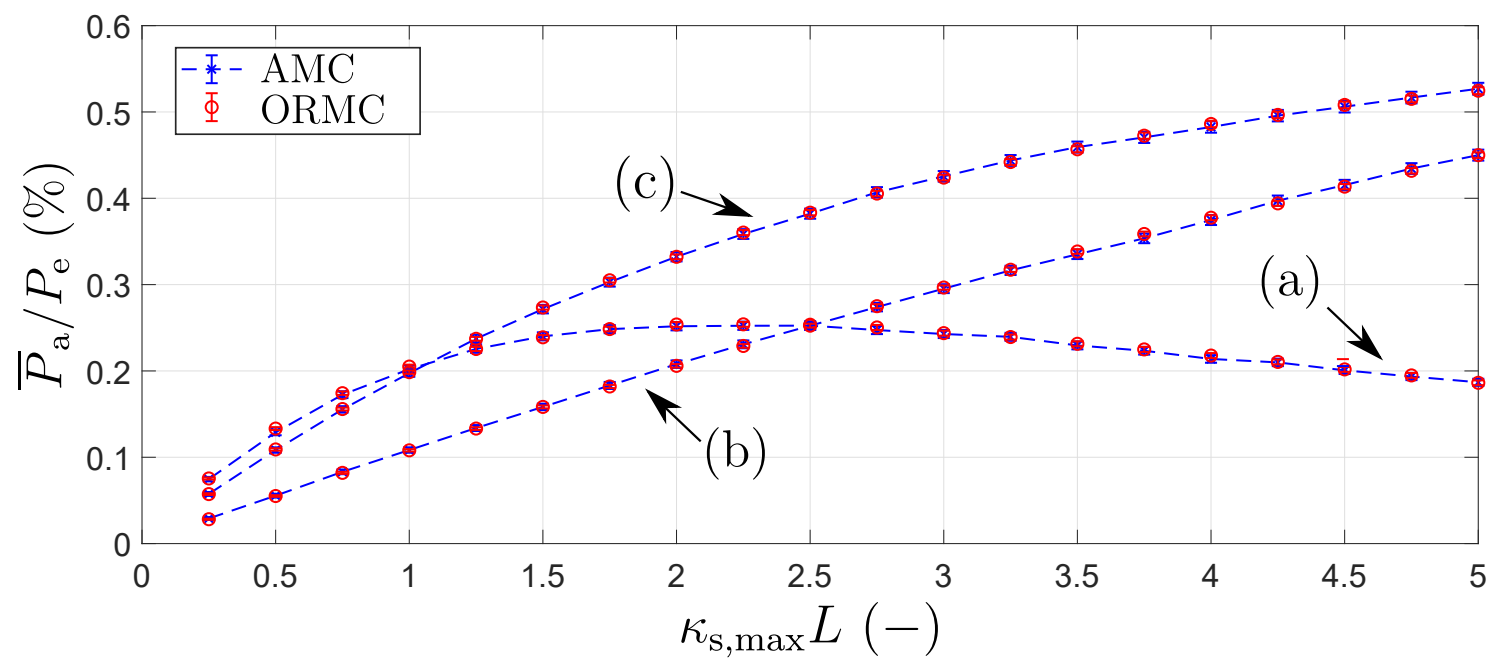

Figure 5: Ratio between power absorbed and power emitted $\bar{P}_{\mathrm{a}} / P_{\mathrm{e}} \pm 3 \bar{\sigma} / P_{\mathrm{e}}$

as a function of maximum scattering optical thickness $\kappa_{\mathrm{s}, \max } L$ for $\kappa_{\mathrm{a}, \max } L=1.0$

and using $10^{7}$ independent realizations. Comparison between ARM and ORMC

algorithm for: (a) homogeneous medium with NCA and double randomization

technique (b) linear profile (c) profile of an axisymmetric flame. 

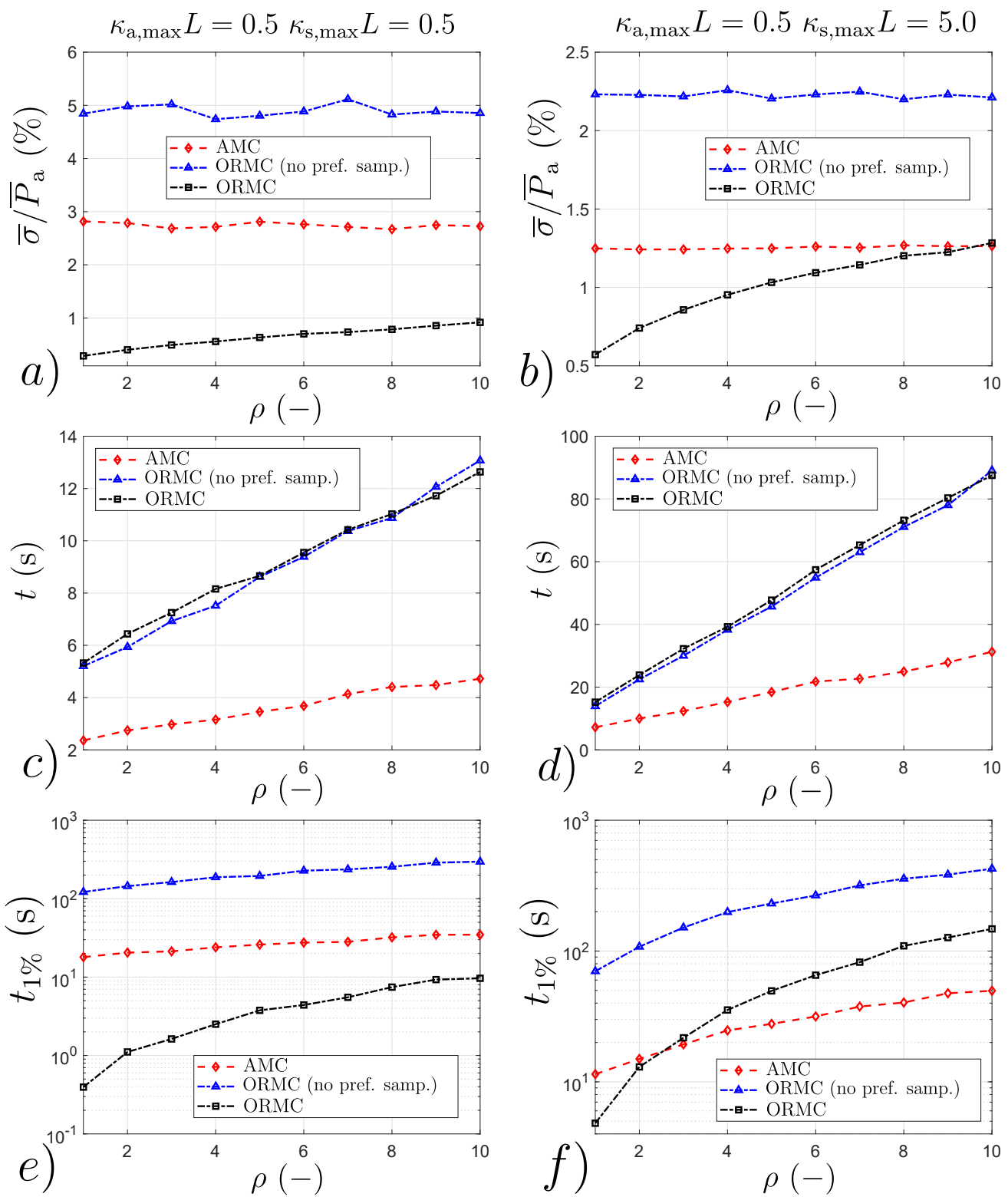

Figure 6: Computational performance of Monte Carlo methods such as Analogous Monte Carlo and Optimized Reverse Monte Carlo (with and without preferential sampling) as a function of the choice of the majorant $\hat{k}_{\mathrm{s}}=\rho \kappa_{\mathrm{s}, \max }$ in the case of heterogeneous medium (axisymmetric flame): a), c) and e) correspond to a slightly scattering medium $\kappa_{\mathrm{s}, \max } L=0.5$ while b), d) and f) correspond to a strongly scattering medium $\kappa_{\mathrm{s}, \max } L=5.0$; a) and b): Relative statistical uncertainty for $10^{6}$ independent realizations. Convergence of ORMC is dependent on the choice of the upper-bound; c) and d) Computation time $t$ for $10^{6}$ independent realization. Based on several sub-paths, ORMC is more sensitive than AMC to the choice of the upper-bound; e) and f) Computation time $t_{1 \%}$ for a $1 \%$ statistical uncertainty. This computation was performed with an "Intel i5 - 2.4 GHz" CPU without any parallelization. 


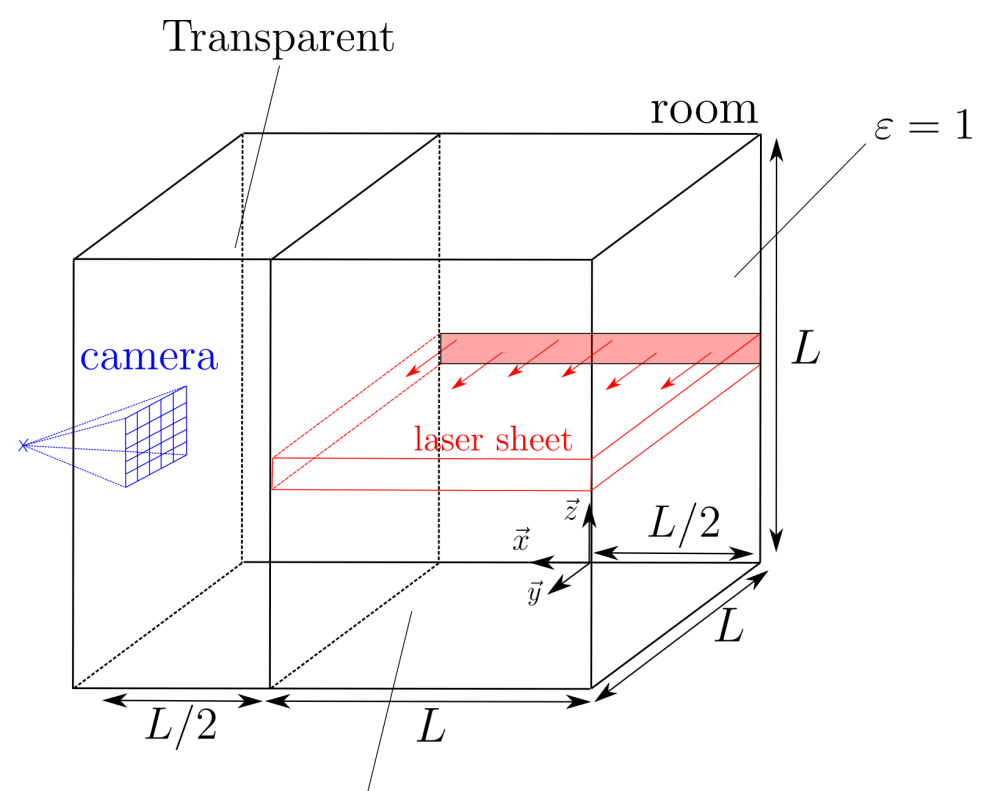

Analytical Profile : $\kappa_{\mathrm{a}}(\mathbf{x}) \kappa_{\mathrm{s}}(\mathbf{x})$

homogeneous

$\kappa_{\mathrm{a}}(\mathbf{x})=\kappa_{\mathrm{a}, \max }$

$\begin{aligned} & \text { heterogeneous (linear) } \\ & \kappa_{\mathrm{s}}(\mathbf{x})=\kappa_{\mathrm{s}, \max }\left(\frac{L / 2-x}{L}\right)\left(\frac{y}{L}\right)\left(\frac{z}{L}\right)\end{aligned} \mid \begin{aligned} & \kappa_{\mathrm{a}, \max }\left(\frac{L / 2-x}{L}\right)\left(\frac{y}{L}\right)\left(\frac{z}{L}\right) \\ & \kappa_{\mathrm{a}}(\mathbf{x})=\kappa_{\mathrm{a}, \max }\left(\frac{z}{L}\right)\left(1-\sqrt{\frac{\left(x-x_{c}\right)^{2}+\left(y-y_{c}\right)^{2}}{R_{f}^{2}}}\right) \\ & \kappa_{\mathrm{s}}(\mathbf{x})=\kappa_{\mathrm{s}, \max }\left(\frac{z}{L}\right)\left(1-\sqrt{\frac{\left(x-x_{c}\right)^{2}+\left(y-y_{c}\right)^{2}}{R_{f}^{2}}}\right) \\ & x_{c}=0 \quad y_{c}=L / 2 \quad R_{f}=L / 2\end{aligned}$

Figure 7: Configuration for the computer-generated images with analytical profiles of radiative properties 


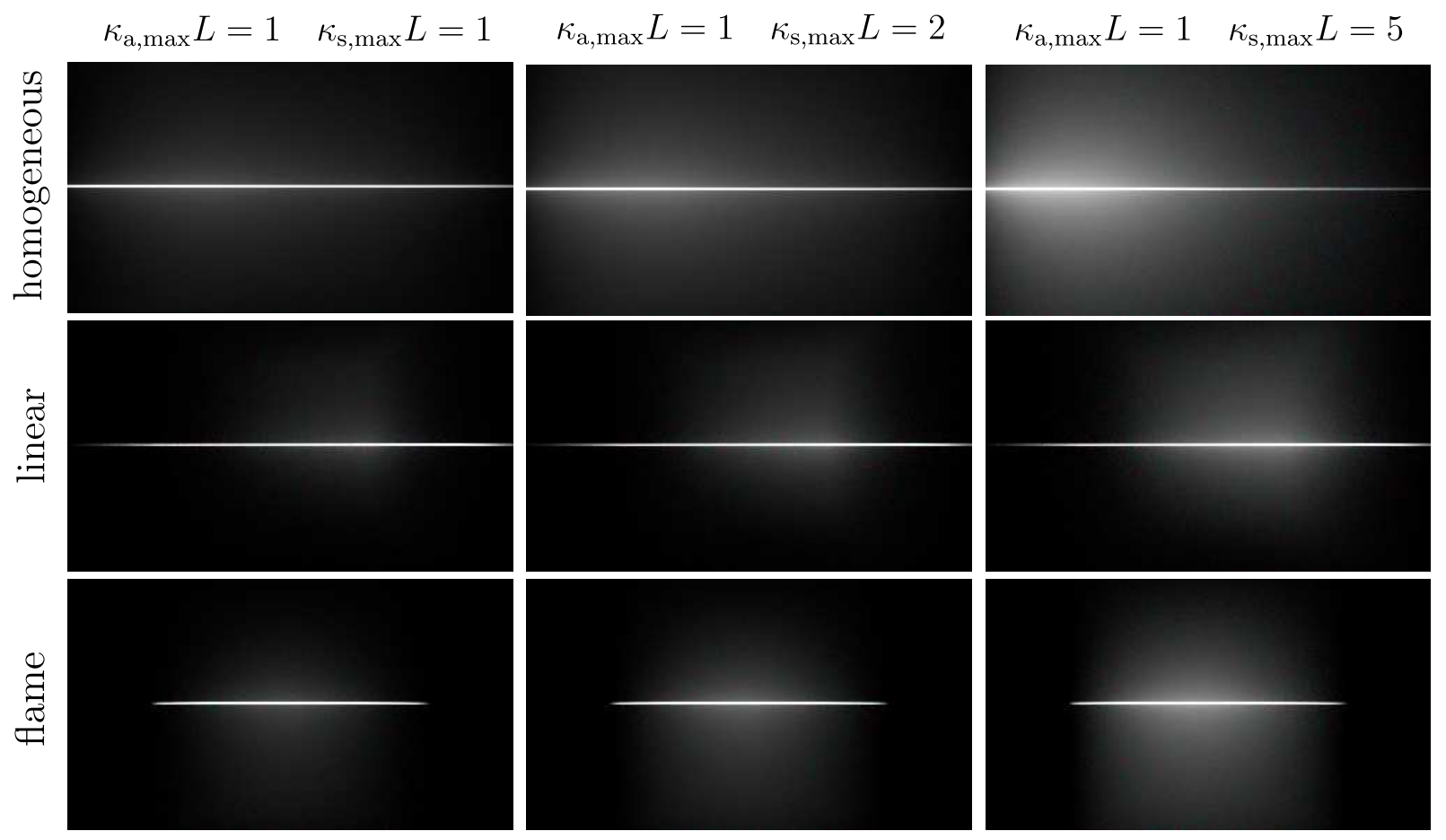

Figure 8: Benchmark images $(320 \times 180$ pixels $)$ of three different analytical profiles: homogeneous medium, linear profile and simili flame profile and for

three different maximum scattering optical thicknesses. Maximum absorption optical thickness is unitary and 30000 realizations per pixel are performed 


\section{References}

[1] R. P. Feynman, A. R. Hibbs, Quantum Mechanics and Path Integrals, McGraw-Hill, New York, 1965.

[2] M. Kac, On distributions of certain wiener functionals, Transactions of the American Mathematical Society 65 (1) (1949) 1-13.

[3] E. Woodcock, T. Murphy, P. Hemmings, S. Longworth, Techniques used in the gem code for monte carlo neutronics calculations in reactors and other systems of complex geometry, in: Proc. Conf. Applications of Computing Methods to Reactor Problems, Vol. 557, 1965.

[4] J. Novák, I. Georgiev, J. Hanika, W. Jarosz, Monte carlo methods for volumetric light transport simulation, in: Computer Graphics Forum, Vol. 37, Wiley Online Library, 2018, pp. 551-576.

[5] G. I. Marchuk, G. A. Mikhailov, M. A. Nazaraliev, R. A. Darbinjan, B. A Kargin, B. S. Elepov, Elements of Radiative-Transfer Theory Used in the Monte Carlo Methods, in: The Monte Carlo Methods in Atmospheric Optics, Springer Series in Optical Sciences, Springer, Berlin, Heidelberg, 1980, pp. 5-17, dOI:10.1007/978-3-540-35237-2_2.

[6] M. Galtier, S. Blanco, C. Caliot, C. Coustet, J. Dauchet, M. El Hafi, V. Eymet, R. Fournier, J. Gautrais, A. Khuong, et al., Integral formulation of null-collision monte carlo algorithms, Journal of Quantitative Spectroscopy and Radiative Transfer 125 (2013) 57-68.

[7] V. Eymet, D. Poitou, M. Galtier, M. El Hafi, G. Terrée, R. Fournier, Nullcollision meshless monte-carlo-application to the validation of fast radiative transfer solvers embedded in combustion simulators, Journal of Quantitative Spectroscopy and Radiative Transfer 129 (2013) 145-157.

[8] J. Dauchet, J.-J. Bézian, S. Blanco, C. Caliot, J. Charon, C. Coustet, M. El Hafi, V. Eymet, O. Farges, V. Forest, et al., Addressing nonlinearities in monte carlo, Scientific reports 8 (1) (2018) 13302.

[9] M. E. Hafi, S. Blanco, J. Dauchet, R. Fournier, M. Galtier, L. Ibarrart, J.M. Tregan, N. Villefranque, Three viewpoints on null-collision Monte Carlo algorithms, Journal of Quantitative Spectroscopy and Radiative Transfer (2020).

[10] N. Villefranque, R. Fournier, F. Couvreux, S. Blanco, C. Cornet, V. Eymet, V. Forest, J.-M. Tregan, A path-tracing monte carlo library for 3 -d radiative transfer in highly resolved cloudy atmospheres, Journal of Advances in Modeling Earth Systemsdoi: 10.1029/2018MS001602.

[11] W. L. Dunn, J. K. Shultis, Exploring monte carlo methods, Elsevier, 2011

[12] R. R. Coveyou, V. Cain, K. Yost, Adjoint and importance in monte carlo application, Nuclear Science and Engineering 27 (2) (1967) 219-234.

[13] K. Daun, K. Thomson, F. Liu, Simulation of laser-induced incandescence measurements in an anisotropically scattering aerosol through backward monte carlo, Journal of heat transfer 130 (11) (2008).

[14] E. Veach, Robust Monte Carlo methods for light transport simulation, Vol. 1610, Stanford University PhD thesis, 1997.

[15] M. F. Modest, Radiative heat transfer, Academic press, 2013.

[16] B. T. Wong, M. P. Mengüç, Comparison of monte carlo techniques to predict the propagation of a collimated beam in participating media, $\mathrm{Nu}-$ merical Heat Transfer: Part B: Fundamentals 42 (2) (2002) 119-140.

[17] J. Dauchet, J.-J. Bezian, S. Blanco, C. Caliot, J. Charon, C. Coustet, M. El Hafi, V. Eymet, O. Farges, V. Forest, R. Fournier, M. Galtier, J. Gautrais, A. Khuong, L. Pelissier, B. Piaud, M. Roger, G. Terrée, S. Weitz, Addressing nonlinearities in Monte Carlo, Scientific reports 8 (2018) 20452322. doi:10.1038/s41598-018-31574-4.

[18] A. De Lataillade, J. Dufresne, M. El Hafi, V. Eymet, R. Fournier, A netexchange monte carlo approach to radiation in optically thick systems, Journal of Quantitative Spectroscopy and Radiative Transfer 74 (5) (2002) 563-584.

[19] D. Ramon, F. Steinmetz, D. Jolivet, M. Compiègne, R. Frouin, Modeling polarized radiative transfer in the ocean-atmosphere system with the gpu-accelerated smart-g monte carlo code, Journal of Quantitative Spectroscopy and Radiative Transfer 222 (2019) 89-107. 